\title{
Delineating the Role of Aedes aegypti ABC Transporter Gene Family during Mosquito Development and Arboviral Infection via Transcriptome Analyses
}

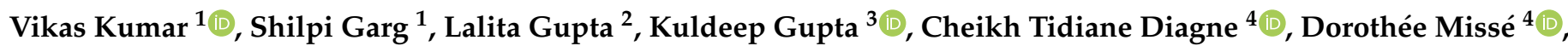 \\ Julien Pompon ${ }^{4}{ }^{\mathbb{D}}$, Sanjeev Kumar ${ }^{5, *,+}$ and Vishal Saxena ${ }^{1, *, \dagger}$ \\ 1 Department of Biological Sciences, Birla Institute of Technology and Science (BITS), Pilani Campus, \\ Pilani 333031, India; vlamba13@gmail.com or p2016019@pilani.bits-pilani.ac.in (V.K.); \\ shilpi@pilani.bits-pilani.ac.in (S.G.) \\ 2 Department of Zoology, Chaudhary Bansi Lal University, Bhiwani 127021, India; lalitagupta@yahoo.com \\ or lalita.zoology@cblu.ac.in \\ 3 Department of Radiology and Radiological Sciences, School of Medicine, 470 Cancer Research Building-II, \\ Johns Hopkins University, Baltimore, MD 21218, USA; kgupta14@jhmi.edu \\ 4 MIVEGEC, Univ. Montpellier, IRD, CNRS, 34394 Montpellier, France; tidiane.diagne@ird.fr (C.T.D.); \\ dorothee.misse@ird.fr (D.M.); julien.pompon@ird.fr (J.P.) \\ 5 Department of Biotechnology, Chaudhary Bansi Lal University, Bhiwani 127021, India \\ * Correspondence: sanjeevnih@gmail.com or hodbiotech@cblu.ac.in (S.K.); \\ vishalsaxena@pilani.bits-pilani.ac.in (V.S.) \\ + Both the authors contributed equally.
}

Citation: Kumar, V.; Garg, S.; Gupta, L.; Gupta, K.; Diagne, C.T.; Missé, D.; Pompon, J.; Kumar, S.; Saxena, V. Delineating the Role of Aedes aegypti ABC Transporter Gene Family during Mosquito Development and Arboviral Infection via Transcriptome Analyses. Pathogens 2021, 10, 1127. https://doi.org/10.3390/pathogens 10091127

Academic Editor: Stephanie

L. Richards

Received: 31 July 2021

Accepted: 30 August 2021

Published: 2 September 2021

Publisher's Note: MDPI stays neutral with regard to jurisdictional claims in published maps and institutional affiliations.

Copyright: () 2021 by the authors Licensee MDPI, Basel, Switzerland. This article is an open access article distributed under the terms and conditions of the Creative Commons Attribution (CC BY) license (https:// creativecommons.org/licenses/by/ $4.0 /)$.
Abstract: Aedes aegypti acts as a vector for several arboviral diseases that impose a major socioeconomic burden. Moreover, the absence of a vaccine against these diseases and drug resistance in mosquitoes necessitates the development of new control strategies for vector-borne diseases. ABC transporters that play a vital role in immunity and other cellular processes in different organisms may act as non-canonical immune molecules against arboviruses, however, their role in mosquito immunity remains unexplored. This study comprehensively analyzed various genetic features of putative $\mathrm{ABC}$ transporters and classified them into A-H subfamilies based on their evolutionary relationships. Existing RNA-sequencing data analysis indicated higher expression of cytosolic ABC transporter genes (E \& F Subfamily) throughout the mosquito development, while members of other subfamilies exhibited tissue and time-specific expression. Furthermore, comparative gene expression analysis from the microarray dataset of mosquito infected with dengue, yellow fever and West Nile viruses revealed 31 commonly expressed ABC transporters suggesting a potentially conserved transcriptomic signature of arboviral infection. Among these, only a few transporters of ABCA, $\mathrm{ABCC}$ and $\mathrm{ABCF}$ subfamily were upregulated, while most were downregulated. This indicates the possible involvement of $\mathrm{ABC}$ transporters in mosquito immunity.

Keywords: Aedes aegypti; ABC transporters; immunity; arboviruses; dengue virus

\section{Introduction}

The Aedes aegypti (Ae. aegypti) mosquito transmits a wide range of arboviruses such as dengue (DENV), Chikungunya (CHIKV), West Nile (WNV), yellow fever (YFV) and Zika virus (ZIKV) that are known to cause severe health problems in human. Among these, DENV is one of the most dangerous infections, which inflicts over 390 million individuals annually with nearly half of the world population at the risk of infection [1,2]. The large human population, expanding urbanization, a significant increase in trade as well as tourism, and climate change are the major contributing factors to augment the disease transmission [3]. Various efforts to eliminate or control the mosquito vectors have faced a significant setback due to the development of insecticide resistance and the unavailability 
of effective disease transmission-blocking vaccines. These facts substantiate a need to understand the mechanisms and factors responsible for the disease transmission and to develop strategies for its control.

The interaction of viruses with the mosquito system is initiated just after their ingestion. They spatially and sequentially interact with different mosquito organs; gut, being the first organ of encounter and salivary glands as the last organ of interaction. Although these organs mount an array of immune responses against pathogens, still, the virus somehow manages to escape the mosquito immunity [4-6]. Many studies have identified critical mosquito molecules that either inhibit or support virus propagation and survival. Notably, the mosquito immune signaling pathways such as Toll, IMD and JAK-STAT are reported to be activated against a wide range of pathogens and are negatively regulated by Cactus, Caspar and PIAS, respectively [7-10]. A recent report also revealed the broad antiviral role of Ae. aegypti JNK pathway [11]. In addition, the mosquito machinery that cleaves viral RNA through RNA interference (RNAi) also exhibits an antiviral response [12,13].

Identification and characterization of non-canonical or novel immune molecules, which may regulate pathogen development might offer new insights to interrupt the mosquito cycle of blood-borne pathogens [14]. ABC transporters, the transmembrane molecular pumps, which utilize ATP to translocate a variety of molecules across the biological membrane have been shown to play an important immune role in some insects $[15,16]$. For example, RNAi-mediated silencing of an ABC transporter sulphonylurea receptor (Sur), which is part of a potassium channel, makes Drosophila hypersensitive to viral infections [15]. In addition, some of the ABCC subfamily transporters are also reported to exhibit a strong negative correlation with Plasmodium infection in Anopheles gambiae [17,18].

ABC transporters share a typical architectural organization comprising highly conserved hydrophilic nucleotide-binding domains (NBDs) and less conserved hydrophobic transmembrane domains (TMDs) [19]. NBDs, also known as ATP-binding cassettes, are located at the cytoplasmic side of the cell membrane and hydrolyze ATP to generate translocation driving energy. They contain various conserved signature motifs like Walker A $(\mathrm{GXXGXGK}(\mathrm{S} / \mathrm{T}))$, Walker B $(\varphi \varphi \varphi \varphi \mathrm{D}$, here $\varphi$ denotes a hydrophobic residue), Q-loop, D-loop, and H-motif, as well as C-motif (LSGGQ). The transmembrane domains (TMDs) form the translocation pathway and provide specificity to a substrate [20-22]. Based on the number or presence/absence of NBDs or TMDs, the ABC transporters have been characterized as full, half or cytosolic transporters. The full ABC transporter contains four functional domains, two NBDs and two TMDs encoded by a single gene. The members that contain one TMD and one NBD, are called half transporters and are functional as homo-or heterodimers [23]. The members that lack the TMDs are termed cytosolic transporters and are mainly regulatory in function. Insect $A B C$ transporters superfamily is further subdivided into eight subfamilies (ABCA to $\mathrm{ABCH}$ ), based on the phylogenetic relationship among their NBDs [22,24].

In this study, we identified and comprehensively analyzed the ABC transporters superfamily from Ae. aegypti genome assembly 5 (AaegL5) [25] through characterization of their conserved domains, structural topology, chromosomal location, and phylogenetic relationship. Further, the expression patterns of these transporters were also analyzed using the available transcriptome datasets to delineate their functions during mosquito development and post arboviral infections [26,27]. The transcriptomic alterations were validated by quantitative real-time PCR (qPCR) for a few representative genes.

\section{Results}

\subsection{Genomic Location and Curated Details of Ae. aegypti ABC Transporters}

We have identified a total of 59 putative ABC transporter genes from Ae. aegypti genome assembly 5 (AaegL5) [25], which is in contrast to previous studies performed by Lu et al. and Figueira-Mansur et al. [26,28] that reported 69 and 53 ABC transporters, respectively from Ae. aegypti genome. These reports included long non-coding RNA, uncharacterized protein, and pseudogenes, which is now curated in the recently updated 
genome assembly (AaegL5) [25]. Our analyses revealed that among 59 transporter genes, 47 genes have expression sequence tags (ESTs) support, while the remaining 12 genes are without ESTs, however, they all showed the evidence of expression in at least one of the analyzed transcriptomic datasets (Table 1).

Table 1. ABC transporters identified from Ae. aegypti genome assembly 5 (AaegL5) [25]. $(+)=$ Sense strand; $(-)=$ Antisense strand; $\mathrm{Y}-\mathrm{Yes}, \mathrm{N}-\mathrm{No}$.

\begin{tabular}{|c|c|c|c|c|c|c|c|c|}
\hline \multirow[b]{2}{*}{$\begin{array}{c}\text { АBC } \\
\text { Transporter } \\
\text { Gene }\end{array}$} & \multirow[b]{2}{*}{$\begin{array}{l}\text { VectorBase } \\
\text { ID }\end{array}$} & \multirow[b]{2}{*}{$\begin{array}{l}\text { Chromosome: Physical } \\
\text { Location (Strand) }\end{array}$} & \multirow[b]{2}{*}{$\begin{array}{c}\text { No. } \\
\text { of } \\
\text { Exons }\end{array}$} & \multirow[b]{2}{*}{$\begin{array}{c}\text { No. of } \\
\text { Transcripts }\end{array}$} & \multirow[b]{2}{*}{$\begin{array}{l}\text { Protein } \\
\text { Length } \\
\text { (AA) }\end{array}$} & \multirow[b]{2}{*}{$\begin{array}{l}\text { Availability } \\
\text { of EST (No. } \\
\text { of ESTs) }\end{array}$} & \multicolumn{2}{|c|}{ Expression Status in } \\
\hline & & & & & & & $\begin{array}{c}\text { Mosquito } \\
\text { Developmental } \\
\text { Stages }\end{array}$ & $\begin{array}{l}\text { Arboviral } \\
\text { Infection }\end{array}$ \\
\hline \multicolumn{9}{|c|}{ ABCA Subfamily (11 Genes) } \\
\hline AaeABCA1 & AAEL012698 & 2:94093797-94103717(-) & 8 & 3 & 1641 & $\mathrm{Y}(4)$ & $\mathrm{N}$ & $\mathrm{Y}$ \\
\hline AaeABCA2.1 & AAEL012702 & 2:94177008-94183151(-) & 11 & 1 & 1636 & Y (26) & $\mathrm{N}$ & $\mathrm{Y}$ \\
\hline AaeABCA2.2 & AAEL012700 & 2:94160180-94166404(-) & 11 & 1 & 1645 & $\mathrm{~N}$ & $\mathrm{~N}$ & $\mathrm{Y}$ \\
\hline AaeABCA4 & AAEL001938 & 3:227108694-227135343(+) & 8 & 1 & 1673 & $\mathrm{Y}(11)$ & $\mathrm{Y}$ & $\mathrm{Y}$ \\
\hline AaeABCA5 & AAEL008386 & 3:224734279-224779573(+) & 9 & 2 & 1663 & $\mathrm{Y}(5)$ & $\mathrm{Y}$ & $\mathrm{Y}$ \\
\hline AaeABCA6 & AAEL008384 & 3:224779816-224802105(+) & 10 & 1 & 1657 & Y (1) & Y & $\mathrm{Y}$ \\
\hline AaeABCA7.1 & AAEL017572 & 1:117261376-117263613(-) & 2 & 1 & 590 & $\mathrm{~N}$ & $\mathrm{Y}$ & $\mathrm{N}$ \\
\hline AaeABCA7.2 & AAEL014699 & 3:51218604-51279389(+) & 7 & 2 & 1635 & Y (18) & $\mathrm{Y}$ & $\mathrm{Y}$ \\
\hline AaeABCA8 & AAEL021738 & 3:365856244-365899503(+) & 12 & 2 & 1900 & $\mathrm{Y}(3)$ & $\mathrm{Y}$ & $\mathrm{Y}$ \\
\hline AaeABCA9 & AAEL018040 & 3:322613800-322714818(-) & 16 & 2 & 1987 & $Y(26)$ & $\mathrm{Y}$ & $\mathrm{Y}$ \\
\hline AaeABCA10 & AAEL012701 & 2:94115322-94145067(-) & 8 & 1 & 1622 & $\mathrm{Y}(6)$ & $\mathrm{N}$ & $\mathrm{Y}$ \\
\hline \multicolumn{9}{|c|}{ ABCB Subfamily (5 Genes) } \\
\hline$A a e A B C B 1$ & AAEL008134 & 3:107404546-107431730(+) & 5 & 1 & 848 & Y (20) & $\mathrm{Y}$ & $\mathrm{Y}$ \\
\hline AaeABCB2 & AAEL010379 & 2:182523007-182607302(+) & 11 & 3 & 1307 & $\mathrm{Y}(2)$ & $\mathrm{Y}$ & $\mathrm{Y}$ \\
\hline АаeАВСВ3 & AAEL022941 & 2:78738879-78757036(-) & 3 & 1 & 725 & Y (10) & $\mathrm{Y}$ & $\mathrm{Y}$ \\
\hline АаeАВCB4 & AAEL006717 & 2:85121437-85146605(+) & 6 & 2 & 820 & Y (9) & Y & $\mathrm{Y}$ \\
\hline AaeABCB5 & AAEL000434 & 3:187161644-187197938(+) & 7 & 1 & 693 & $Y(8)$ & $\mathrm{Y}$ & $\mathrm{Y}$ \\
\hline \multicolumn{9}{|c|}{ ABCC Subfamily (16 Genes) } \\
\hline AaeABCC1 & AAEL005918 & 3:138401412-138418395(-) & 9 & 1 & 1312 & Y (17) & $\mathrm{Y}$ & $\mathrm{Y}$ \\
\hline$A a e A B C C 2$ & AAEL025460 & 3:138444756-138480164(+) & 9 & 4 & 1388 & $\mathrm{Y}(13)$ & $\mathrm{Y}$ & $\mathrm{Y}$ \\
\hline AaeABCC 3 & AAEL005929 & 3:138489236-138524582(+) & 11 & 3 & 1419 & $\mathrm{Y}(7)$ & $\mathrm{Y}$ & $\mathrm{Y}$ \\
\hline AaeABCC4.1 & AAEL012395 & 1:284711177-284717415(+) & 5 & 2 & 1357 & Y (9) & $\mathrm{N}$ & $\mathrm{Y}$ \\
\hline AaeABCC 4.2 & AAEL019847 & 1:284693063-284698462(-) & 6 & 1 & 1355 & $\mathrm{~N}$ & $\mathrm{~N}$ & $\mathrm{Y}$ \\
\hline AaeABCC5 & AAEL023958 & 1:284737104-284863028(+) & 13 & 1 & 1488 & $\mathrm{Y}(4)$ & $\mathrm{N}$ & $\mathrm{Y}$ \\
\hline AaeABCC6 & AAEL027539 & 1:284869084-284903303(+) & 11 & 3 & 1286 & $\mathrm{~N}$ & $\mathrm{~N}$ & $\mathrm{Y}$ \\
\hline AaeABCC7 & AAEL018267 & 2:100234384-100334146(+) & 7 & 6 & 1394 & $Y(23)$ & $\mathrm{Y}$ & $\mathrm{Y}$ \\
\hline AaeABCC 8 & AAEL005045 & 2:445037808-445059189(-) & 5 & 2 & 1508 & $\mathrm{~N}$ & $\mathrm{Y}$ & $\mathrm{Y}$ \\
\hline АаeАВСС9 & AAEL005026 & 2:445021476-445037944(+) & 5 & 1 & 1454 & $\mathrm{~N}$ & $\mathrm{Y}$ & $\mathrm{Y}$ \\
\hline AaeABCC10 & AAEL005043 & 2:444993656-444999015(+) & 6 & 2 & 1522 & $\mathrm{Y}(13)$ & $\mathrm{Y}$ & $\mathrm{Y}$ \\
\hline AaeABCC12 & AAEL020303 & 2:463313906-463367902(+) & 5 & 1 & 1532 & $Y(2)$ & $\mathrm{Y}$ & $\mathrm{Y}$ \\
\hline AaeABCC13 & AAEL023524 & 2:312907331-313134735(+) & 25 & 1 & 2101 & $\mathrm{Y}(1)$ & $\mathrm{Y}$ & $\mathrm{Y}$ \\
\hline AaeABCC14 & AAEL004743 & 2:289194273-289251024(+) & 15 & 15 & 1524 & Y (27) & $\mathrm{Y}$ & $\mathrm{Y}$ \\
\hline AaeABCC16 & AAEL017209 & 3:403021800-403026639(+) & 4 & 1 & 986 & $\mathrm{~N}$ & $\mathrm{Y}$ & $\mathrm{N}$ \\
\hline AaeABCC17 & AAEL015644 & 1:193810018-193840226(+) & 9 & 1 & 1339 & $Y(10)$ & $\mathrm{Y}$ & $\mathrm{Y}$ \\
\hline \multicolumn{9}{|c|}{ ABCD Subfamily (2 Genes) } \\
\hline AaeABCD1 & AAEL010047 & 1:160091832-160259983(+) & 7 & 2 & 753 & $\mathrm{Y}(14)$ & $\mathrm{Y}$ & $\mathrm{Y}$ \\
\hline$A a e A B C D 2$ & AAEL002913 & 1:108683838-108734858(+) & 10 & 1 & 659 & Y (27) & $\mathrm{Y}$ & Y \\
\hline
\end{tabular}


Table 1. Cont.

\begin{tabular}{|c|c|c|c|c|c|c|c|c|}
\hline \multirow[b]{2}{*}{$\begin{array}{c}\text { ABC } \\
\text { Transporter } \\
\text { Gene }\end{array}$} & \multirow[b]{2}{*}{$\begin{array}{l}\text { VectorBase } \\
\text { ID }\end{array}$} & \multirow[b]{2}{*}{$\begin{array}{l}\text { Chromosome: Physical } \\
\text { Location (Strand) }\end{array}$} & \multirow[b]{2}{*}{$\begin{array}{l}\text { No. } \\
\text { of } \\
\text { Exons }\end{array}$} & \multirow[b]{2}{*}{$\begin{array}{c}\text { No. of } \\
\text { Transcripts }\end{array}$} & \multirow[b]{2}{*}{$\begin{array}{l}\text { Protein } \\
\text { Length } \\
\text { (AA) }\end{array}$} & \multirow[b]{2}{*}{$\begin{array}{l}\text { Availability } \\
\text { of EST (No. } \\
\text { of ESTs) }\end{array}$} & \multicolumn{2}{|c|}{ Expression Status in } \\
\hline & & & & & & & $\begin{array}{c}\text { Mosquito } \\
\text { Developmental } \\
\text { Stages }\end{array}$ & $\begin{array}{l}\text { Arboviral } \\
\text { Infection }\end{array}$ \\
\hline \multicolumn{9}{|c|}{ ABCE Subfamily (1 Gene) } \\
\hline AaeABCE1 & AAEL010059 & 3:11225514-11239622(+) & 5 & 1 & 609 & Y (66) & $\mathrm{Y}$ & $\mathrm{Y}$ \\
\hline \multicolumn{9}{|c|}{ ABCF Subfamily (3 Genes) } \\
\hline AaeABCF1 & AAEL001101 & 3:406460452-406484817(-) & 2 & 1 & 894 & $\mathrm{Y}(16)$ & Y & $\mathrm{Y}$ \\
\hline AaeABCF2 & AAEL010977 & 3:190430154-190451030(+) & 4 & 2 & 602 & Y (97) & $\mathrm{Y}$ & Y \\
\hline AaeABCF3 & AAEL010359 & 3:372039893-372056253(+) & 5 & 1 & 712 & Y (52) & Y & Y \\
\hline \multicolumn{9}{|c|}{ ABCG Subfamily (18 Genes) } \\
\hline AaeABCG1 & AAEL016999 & 1:107942728-108005678(-) & 8 & 3 & 716 & $\mathrm{Y}(17)$ & Y & $\mathrm{N}$ \\
\hline AaeABCG2 & AAEL021570 & 1:30489715-30492114(-) & 4 & 1 & 687 & $\mathrm{~N}$ & Y & $\mathrm{N}$ \\
\hline AaeABCG3 & AAEL008138 & 3:79113447-79286804(-) & 8 & 5 & 862 & Y (15) & $\mathrm{Y}$ & $\mathrm{Y}$ \\
\hline AaeABCG4 & AAEL003703 & 3:65749828-65763092(-) & 6 & 1 & 616 & Y (1) & $\mathrm{Y}$ & $\mathrm{Y}$ \\
\hline AaeABCG5 & AAEL017188 & 2:24405169-24438842(+) & 8 & 1 & 614 & $\mathrm{~N}$ & $\mathrm{Y}$ & $\mathrm{N}$ \\
\hline AaeABCG7 & AAEL008672 & 2:239556260-239647710(-) & 7 & 2 & 699 & Y (29) & $\mathrm{Y}$ & $\mathrm{Y}$ \\
\hline AaeABCG8 & AAEL019463 & 2:455430514-455514073(+) & 7 & 1 & 723 & Y (10) & Y & Y \\
\hline AaeABCG10 & AAEL027367 & 2:455272483-455402105(+) & 11 & 7 & 726 & Y (6) & Y & Y \\
\hline AaeABCG11 & AAEL008635 & 2:455184210-455236417(+) & 9 & 1 & 676 & Y (17) & Y & Y \\
\hline AaeABCG13 & AAEL022734 & 1:215452060-215689779(-) & 10 & 5 & 888 & $Y(1)$ & $\mathrm{Y}$ & $\mathrm{Y}$ \\
\hline AaeABCG14 & AAEL027424 & 1:215707794-215774030(+) & 6 & 1 & 602 & $\mathrm{Y}(12)$ & $\mathrm{Y}$ & $\mathrm{Y}$ \\
\hline AaeABCG15 & AAEL019641 & 2:270847147-271027814(-) & 12 & 3 & 604 & $Y(8)$ & $\mathrm{Y}$ & $\mathrm{Y}$ \\
\hline AaeABCG16 & AAEL008625 & 2:455514069-455540158(-) & 5 & 1 & 606 & $\mathrm{~N}$ & $\mathrm{Y}$ & $\mathrm{Y}$ \\
\hline AaeABCG17 & AAEL008628 & 2:455557594-455572391(+) & 6 & 2 & 598 & $\mathrm{~N}$ & Y & $\mathrm{Y}$ \\
\hline AaeABCG18 & AAEL008632 & 2:455591457-455609699(+) & 6 & 3 & 607 & $Y(4)$ & $\mathrm{Y}$ & Y \\
\hline $\begin{array}{c}\text { AaeABCG19/ } \\
\text { G20 }\end{array}$ & AAEL026976 & 2:455663955-455680847(+) & 7 & 3 & 599 & $\mathrm{~N}$ & $\mathrm{Y}$ & $\mathrm{Y}$ \\
\hline AaeABCG21 & AAEL008624 & 2:455615233-455650540(+) & 6 & 5 & 593 & Y (6) & $\mathrm{Y}$ & Y \\
\hline AaeABCG22 & AAEL027686 & 3:223544987-223689078(-) & 8 & 1 & 612 & $\mathrm{Y}(12)$ & $\mathrm{Y}$ & $\mathrm{Y}$ \\
\hline \multicolumn{9}{|c|}{ ABCH Subfamily (3 Genes) } \\
\hline AaeABCH1 & AAEL005491 & 3:59371559-59533418(+) & 8 & 5 & 814 & $Y(1)$ & $\mathrm{Y}$ & $\mathrm{Y}$ \\
\hline АaeABCH2 & AAEL018334 & 1:181178199-181420930(+) & 11 & 5 & 814 & Y (3) & Y & Y \\
\hline АаeАВСНЗ & AAEL014428 & 1:159149149-159331370(-) & 12 & 3 & 727 & $Y(1)$ & $\mathrm{Y}$ & $\mathrm{Y}$ \\
\hline
\end{tabular}

Based on the An. gambiae transporters classification [27], Ae. aegypti ABC transporters were also classified into $\mathrm{ABCA}$ to $\mathrm{ABCH}$ subfamilies (Figure $1 \mathrm{~A}, \mathrm{~B})$. These transporters were further classified into full, half, or cytosolic transporters based on their structural topology (Figure $1 \mathrm{~B}$ ). The $\mathrm{ABCA}, \mathrm{ABCB}$, and $\mathrm{ABCC}$ subfamilies consist of ten, one, and fifteen full transporter genes, respectively; while the $\mathrm{ABCD}, \mathrm{ABCG}$, and $\mathrm{ABCH}$ subfamilies have only half transporters. The $\mathrm{ABCE}$ and $\mathrm{ABCF}$ subfamilies contain cytosolic transporters and do not have TMDs (Figure 1A,B). 
A

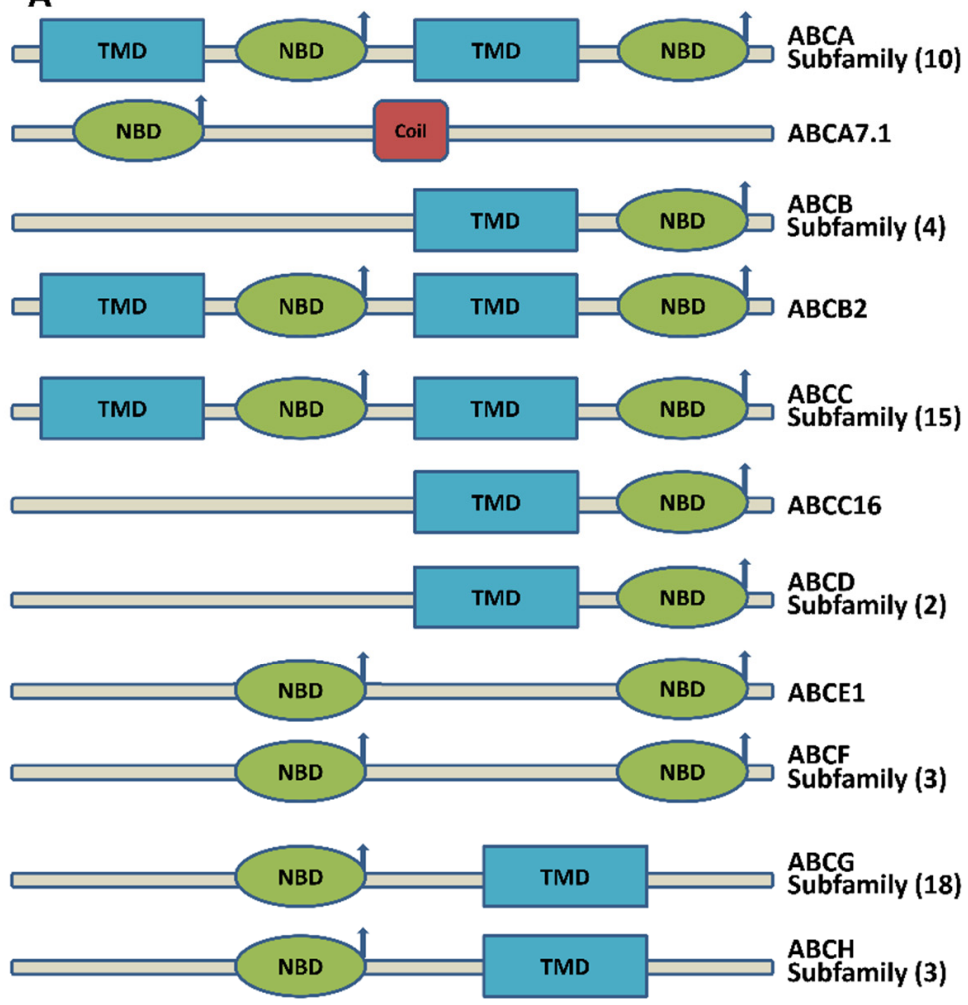

B

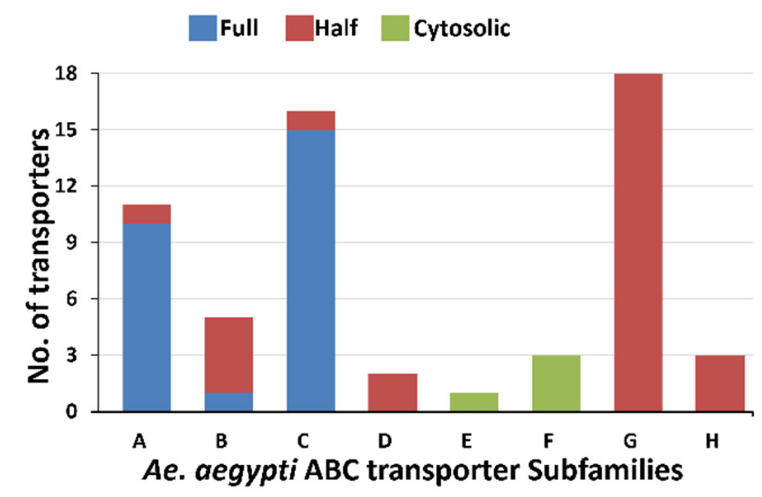

C

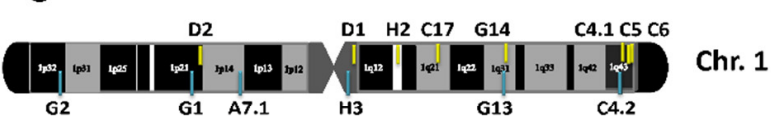

C10 C9 G11 G10 G8 G17 G18 G21 G19 C12

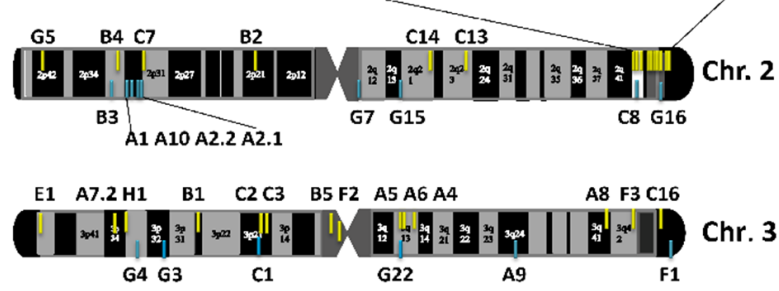

Figure 1. (A) Structural topology of Ae. aegypti ABC transporters. The subfamilies of the ABC transporters are marked on the right-hand side of the structure and the total number of transporters with similar structures in the subfamily are denoted in parenthesis. The arrow denotes the active site (ATPase activity). The transporter ABCA7.1 exclusively has the coiled-coil domain (denoted by the red square). The position of NBD and TMD domains of each Ae. aegypti ABC transporter was identified using the EBI program "phmmer" [29]. (B) Subfamily-wise distribution of full, half and cytosolic $\mathrm{ABC}$ transporters represented by blue, red and green color, respectively. (C) Chromosomal distribution of Ae. aegypti $\mathrm{ABC}$ transporter genes. The physical location of $A B C$ transporter genes is represented in yellow (sense strand) and blue (antisense strand), and the corresponding gene name, in short, is mentioned on the top or bottom side of the individual chromosome.

Chromosome Mapping of $\mathrm{ABC}$ Transporter Genes

Chromosomal mapping revealed that the identified $59 \mathrm{ABC}$ transporter genes are located on three different chromosomes (Figure 1C). Among these, $23.73 \%$ of total ABC transporter genes (14 genes) are located on chromosome 1 . The genes located on chromosome 1 belong to $\mathrm{ABCA}(1 / 11$ members), $\mathrm{ABCC}$ (5/16 members), $\mathrm{ABCD}(2 / 2$ members), $\mathrm{ABCG}(4 / 18$ members) and $\mathrm{ABCH}(2 / 3$ members) subfamilies. $42.37 \%$ of total $\mathrm{ABC}$ transporter genes (25 genes) are located on chromosome 2 . These include clusters of ABCC (6/16 members) and ABCG (10/18 members) located on the long arm, while a cluster of ABCA (4/11 members), ABCB (3/5 members), ABCC (1/16 members) and ABCG $(1 / 18$ members) is located on the short arm of chromosome 2 . Chromosome 3 has remaining $33.9 \%$ of total $A B C$ transporter genes (20 genes) from different $A B C$ subfamilies, including $\mathrm{ABCA}(6 / 11$ members), $\mathrm{ABCB}(2 / 5$ members), $\mathrm{ABCC}(4 / 16$ members), $\mathrm{ABCE}$ (1/1 members), $\mathrm{ABCF}$ (all 3 members), $\mathrm{ABCG}$ ( $3 / 18$ members), and $\mathrm{ABCH}$ ( $1 / 3$ members).

In the comparison of Ae. aegypti $\mathrm{ABC}$ transporters with other reported insect species (such as Drosophila melanogaster [24], An. gambiae [27], Bombyx mori [30], Tribolium castaneum [31], Anopheles sinensis [32] and Culex quinquefasciatus [26]), it was observed that most of the $\mathrm{ABC}$ transporters belong to the $\mathrm{ABCA}, \mathrm{ABCC}$, and $\mathrm{ABCG}$ subfamilies. The $\mathrm{ABCE}$ is the smallest subfamily with only a single member (Table 2). The $A B C D, A B C F$ and $A B C H$ had a similar number of $\mathrm{ABC}$ transporters in all insects. 
Table 2. Subfamily-wise gene number comparison of Ae. aegypti ABC transporters with other organisms.

\begin{tabular}{|c|c|c|c|c|c|c|c|}
\hline $\begin{array}{l}\text { ABC Transporter } \\
\text { Subfamilies }\end{array}$ & $\begin{array}{l}\text { Homo } \\
\text { sapiens }\end{array}$ & $\begin{array}{c}\text { Drosophila } \\
\text { melanogaster }\end{array}$ & $\begin{array}{l}\text { Anopheles } \\
\text { gambiae }\end{array}$ & $\begin{array}{l}\text { Aedes } \\
\text { aegypti }\end{array}$ & $\begin{array}{c}\text { Culex } \\
\text { quinquefasciatus }\end{array}$ & $\begin{array}{l}\text { Tribolium } \\
\text { castaneum }\end{array}$ & $\begin{array}{c}\text { Bombyx } \\
\text { mori }\end{array}$ \\
\hline A & 12 & 10 & 9 & 11 & 10 & 10 & 6 \\
\hline B & 11 & 8 & 5 & 5 & 5 & 6 & 8 \\
\hline C & 12 & 14 & 14 & 16 & 18 & 35 & 15 \\
\hline D & 4 & 2 & 2 & 2 & 2 & 2 & 2 \\
\hline $\mathrm{E}$ & 1 & 1 & 1 & 1 & 1 & 1 & 1 \\
\hline$F$ & 3 & 3 & 3 & 3 & 3 & 3 & 3 \\
\hline G & 5 & 15 & 18 & 18 & 28 & 13 & 13 \\
\hline $\mathbf{H}$ & 0 & 3 & 3 & 3 & 3 & 3 & 3 \\
\hline Total & 48 & 56 & 55 & 59 & 70 & 73 & 51 \\
\hline
\end{tabular}

\subsection{Phylogenetic Analyses and Characteristics of Ae. aegypti ABC Transporters}

Phylogenetic trees were built to categorize the Ae. aegypti $\mathrm{ABC}$ transporters into different subfamilies (Figure 2) and to understand the evolutionary pattern of Ae. aegypti $\mathrm{ABC}$ transporters with orthologues from other insects (Supplementary Figure S1). When compared to the reference genome of An. gambiae, we observed gene duplication and addition of new members indicating genome expansion in Ae. aegypti. The gene duplication was evident in ABCA, ABCC and ABCG subfamilies. Maximum subfamilies exhibited similar branch length indicating common evolutionary distance.

\subsubsection{ABCA Subfamily}

Ae. aegypti has eleven putative members in the ABCA subfamily, all of which are full transporters except AaeABCA7.2, which is a half transporter. A 1:1 orthologous relation was observed among most of the members of AaeABCA subfamily with those from other insects analyzed in this study. There were duplications of transporters such as AaeABCA2.1 and AaeABCA2.2 as well as AaeABCA7.1 and AaeABCA7.2, that are homologous to $A n$. gambiae AGAP006380 ( $A B C A 2)$ and AGAP001523 ( $A B C A 7)$, respectively. Interestingly, the duplication of $A a e A B C A 7$ transporter was observed only in Ae. aegypti. These duplicated genes formed a common clade with $100 \%$ bootstrap support (Supplementary Figure S1A). An additional member of the ABCA subfamily, AaeABCA10 was identified in the Aedes genome, which was not observed in An. gambiae and other insects. Although this gene branches within the ABCA phylogeny group, it appeared as a distant clade. We could not identify any ortholog in the AaegL5 for $A B C A 3$ transporter of $A n$. gambiae and $C x$. quinquefasciatus. The transporters from Ae. aegypti $\mathrm{ABCA}$ subfamily varied from 1773 to 7298 amino acids in length. Among the Aedes ABC transporters, the ABCA subfamily members clustered adjacent to ABCG subfamily in a common clade. A detailed phylogeny of ABCA subfamily members revealed that Drosophila ABCA transporters are present at the base of each clade followed by An. gambiae homologs and Aedes ABCA transporters branching at the end of each clade. 


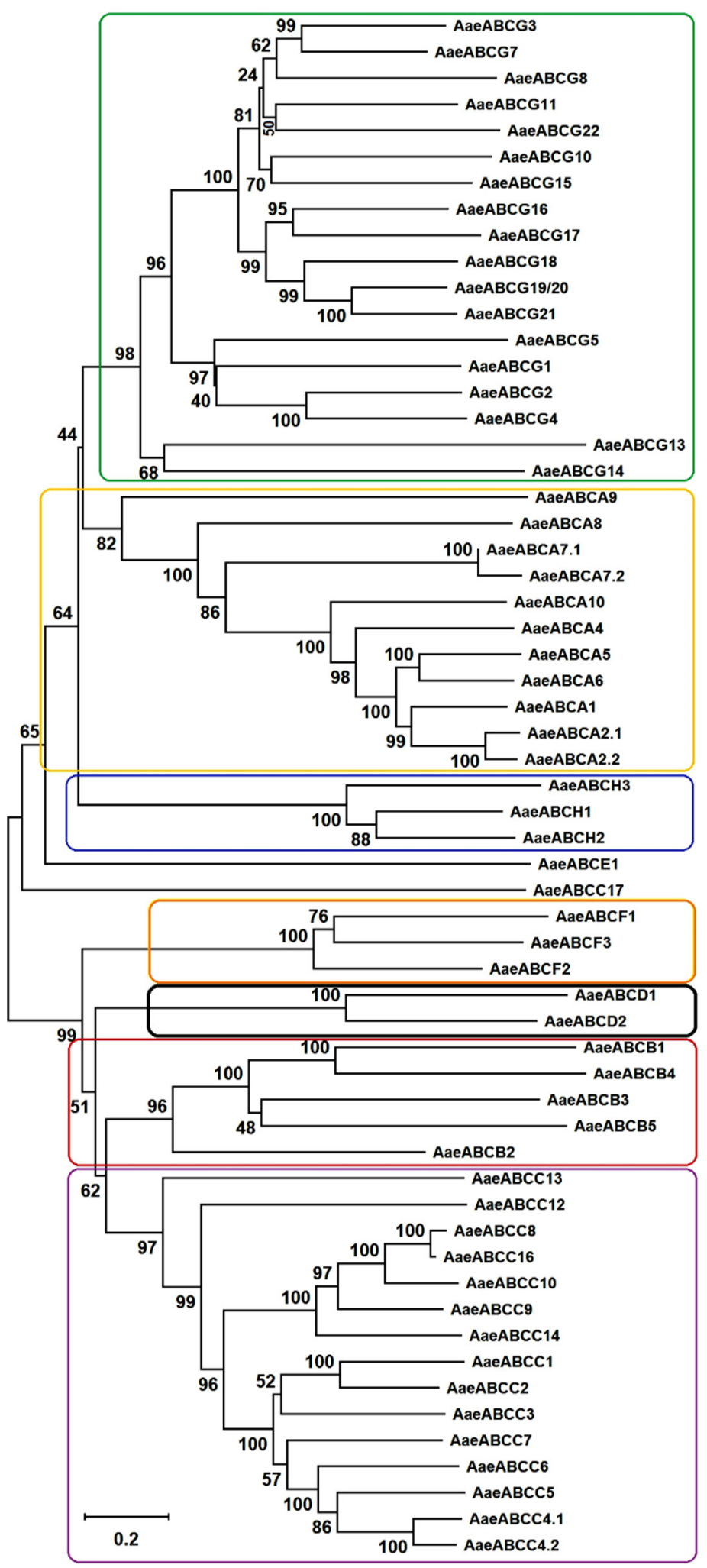

Figure 2. Phylogenetic relationship of Ae. aegypti ABC transporters inferred with the NeighborJoining method using MEGA X [33]. The bootstrap value calculated from 1000 replicates is marked on each corresponding node. The members of each subfamily are enclosed in a different color box except for $A B C E 1$ (a single member of the subfamily) and $A B C C 17$ (falling apart from its other subfamily members). 


\subsubsection{ABCB Subfamily}

We identified five Ae. aegypti $\mathrm{ABCB}$ subfamily members that share a 1:1 relationship with other insect orthologs. Interestingly, no gene duplication and addition/deletion could be identified in this subfamily. Ae. aegypti ABCB subfamily formed a common clade with $\mathrm{ABCC}$ and $\mathrm{ABCD}$ subfamilies (Figure 2). All of these are members of mitochondrial ABC systems and are known to play roles in the iron metabolism and $\mathrm{Fe} / \mathrm{S}$ protein precursors transport [34]. Upon sequence analysis, $A a e A B C B 1$ showed sequence conservation with $A a e A B C B 4$ while $A a e A B C B 3$ showed similarity with $A a e A B C B 5$. Upregulation of Ae. aegypti $A B C B 4$ is reported in a pyrethroid-resistant strain [35]. It is of note that all the members of $A$ e. aegypti $A B C B$ subfamily are half transporters except $A B C B 2$. Ae. aegypti $A B C B 2$ is homologous to D. melanogaster biochemical defense genes (DmMdr49, DmMdr50, and DmMdr65) and An. gambiae ABCB2 (AGAP005639) (Supplementary Figure S1B). AaeABCB2 is a full transporter known as P-glycoprotein, which is linked to insecticide resistance in D. melanogaster and other arthropods [22,36] and has been reported to exhibit a detoxification effect against the insecticide temephos in Ae. aegypti larvae [16].

\subsubsection{ABCC Subfamily}

Ae. aegypti $\mathrm{ABCC}$ transporters constitute the second-largest $\mathrm{ABC}$ subfamily, with 15 full transporters and one-half transporter. All these transporters exhibit 1:1 homology with $A n$. gambiae ABCC members. Interestingly, AaeABCC4 has undergone duplication into AaeABCC4.1 and AaeABCC4.2, and both are located on the opposite strands of the same chromosome. These two duplicates were observed to share high nucleotide similarity $(80 \%)$ with identical introns-exons architecture. We could not identify $A B C C 11$ and $A B C C 15$ members of this subfamily, which are present in An. gambiae, indicating their absence in $A e$. aegypti genome. In the phylogenetic tree, AaeABCC17 formed a cluster with Aedes albopictus and Culex orthologs (Supplementary Figure S1C), but surprisingly it branched away from the rest of the ABCC subfamily cluster and is present adjacent to AaeABCE1 (Figure 2). The appearance of An. gambiae transporter genes at the base of all clusters indicated their possible early origin when compared to Aedes and Culex.

\subsubsection{ABCD Subfamily}

The ABCD subfamily of Ae. aegypti consists of only two half transporters. This subfamily also has a true orthologous relationship with An. gambiae counterparts. Our phylogenetic analyses revealed that $A a A A B C D 1$ clusters with other insect orthologs with $100 \%$ bootstrap support, while AaeABCD2 clusters with its orthologs from other insects with only $71 \%$ bootstrap (Supplementary Figure S1D). These ABC proteins are reported to be involved in fatty acid transport into the peroxisome in insects [37].

\subsubsection{ABCE and ABCF Subfamilies}

Both these subfamilies also show 1:1 orthology with $A B C$ transporters from other insects. Aae $\mathrm{ABCE}$ has only a single member, while Aae $\mathrm{ABCF}$ has three members. Members of both subfamilies show NBD-NBD topology and are devoid of TMDs. Thus, these transporters may not perform any transport function but might play a fundamental role in cellular processes such as translation initiation, elongation, termination, and ribosome biosynthesis etc. as suggested in other organisms [38,39]. AaeABCE1 transporter clusters along with the $A a e \mathrm{ABCA}$ and $A a e \mathrm{ABCH}$ clades; while $A a e \mathrm{ABCF}$ subfamily members clusters with $\mathrm{Aae} \mathrm{ABCB}, \mathrm{Aae} \mathrm{ABCD}$ and $A a e \mathrm{ABCC}$ clades indicating their close evolutionary origin (Figure 2).

\subsection{6. $\mathrm{ABCG}$ and $\mathrm{ABCH}$ Subfamilies}

With eighteen putative members, the ABCG subfamily is the largest transporters subfamily in Ae. aegypti. All its members are half-transporters, like most eukaryotic counterparts except for plants and fungi (which have full transporters). All the Ae. aegypti ABCG subfamily members possess a typical reverse domain architecture (NBD-TMD). Among 
themselves, all the members show high conservation forming a distinct clade upon phylogeny. Most of the AaeABCG transporters show 1:1 orthology with other insects except for An. gambiae ABCG6, which was not identified in Ae. aegypti. AaeABCG19/20 (AAEL026976) is orthologous to both An. gambiae AGAP009471 (ABCG19) and AGAP009472 (ABCG20). We were also able to identify the AaeABCG22 as reported previously [26], which is not identified in An. gambiae (Supplementary Figure S1E). The AaeABCG cluster falls in a clade along with AaeABCA and Aae ABCH subfamilies with $~ 90 \%$ bootstrap, indicating their common evolutionary origin (Figure 2). The AaeABCH subfamily was first identified in $D$. melanogaster and is exclusively reported in insects with no ortholog in mammals, plants, or yeast. Three members of the $\mathrm{ABCH}$ subfamily were identified in Ae. aegypti that have a similar topology of NBDs and TMDs to the AaeABCG transporters, although their function is not yet known.

\subsubsection{ABCJ Subfamily}

An additional $\mathrm{ABC}$ transporter J subfamily was also reported in Ae. aegypti genome, which included relative ABC-ATPases like SMC, Rad50 and MutS [28]. The members of this subfamily are soluble in nature and have a role in DNA repair processes (MutS and Rad50 proteins), and chromosome condensation (SMC) Among these, while Rad50 has a well-conserved LSGG motif, Walker A and B motifs for nucleotide-binding [40], SMCs have more degenerate signature motif and minimal Walker A and B motifs [41]. MutS do not contain signature motif and classical Walker-A [42]. There exist different arguments for the inclusion of these proteins in the $\mathrm{ABC}$ transporter superfamily $[42,43]$. We have also identified an ATP-sensitive $\mathrm{K}^{+}$channel in Ae. aegypti, similar to other organisms [44] that also possess features like $\mathrm{ABC}$ transporters. Thus, more detailed analyses are required for the inclusion of the J subfamily in the ABC superfamily.

\subsection{Expression Profiling of ABC Transporters in Different Developmental Stages of Ae. aegypti}

To delineate and distinguish the importance of $A B C$ transporters, we analyzed their expression profile in different developmental stages (eggs, larvae, pupae and adults) and various organs (carcass and gonads) of mosquito based on the transcriptome data from Akbari et al. [45]. The K-means clustering of all AaeABC transporters, based on their mRNA expression profile was performed (Figure 3) as discussed in Section 4.4. The ABC transporter genes showing high expression patterns (1.5-2.5 $\log _{10}$ expression) from embryo to adult stages, as well as in blood-fed adults, formed a separate cluster (Cluster 1, Figure 3) and included the cytoplasmic subfamily members AaeABCE1, AaeABCF2 and AaeABCF3.

Among the $59 \mathrm{ABC}$ transporters, the expression was only detected for 51 transporters (in at least one of the developmental stages), except for four members each from AaeABCA (AaeABCA1, AaeABCA2.1, AaeABCA2.2 \& AaeABCA10 members) and AaeABCC (AaeABCC4.1, AaeABCC4.2, AaeABCC5 \& AaeABCC6 members) subfamilies. These eight members fall in a distinct cluster (Cluster 2, Figure 3) along with other members exhibiting reduced or minimal expression. Most of the genes in this cluster showed varied expression during different stages except $A a e A B C A 7.1$ and $A a e A B C B 5$ genes that showed a decent expression throughout mosquito development. The genes showing varied expression during specific stages include $A a e A B C A 6$, which showed a high level of expression in NBF ovaries; AaeABCA4, AaeABCG3 and AaeABCH1 that were expressed mainly in carcasses of males with minimal expression in female carcasses (BF and NBF). Of these genes, AaeABCH1 showed good expression during larval and pupal development also while AaeABCG3 exhibits minimal expression during these stages. Aae $A B C A 8$ and $A a e A B C C 9$ showed expression in both male and female carcasses as well as during embryonic development. Few genes that did not show expression in carcass are AaeABCG13 and AaeABCG14, however, they exhibit a high level of expression during embryonic development. 


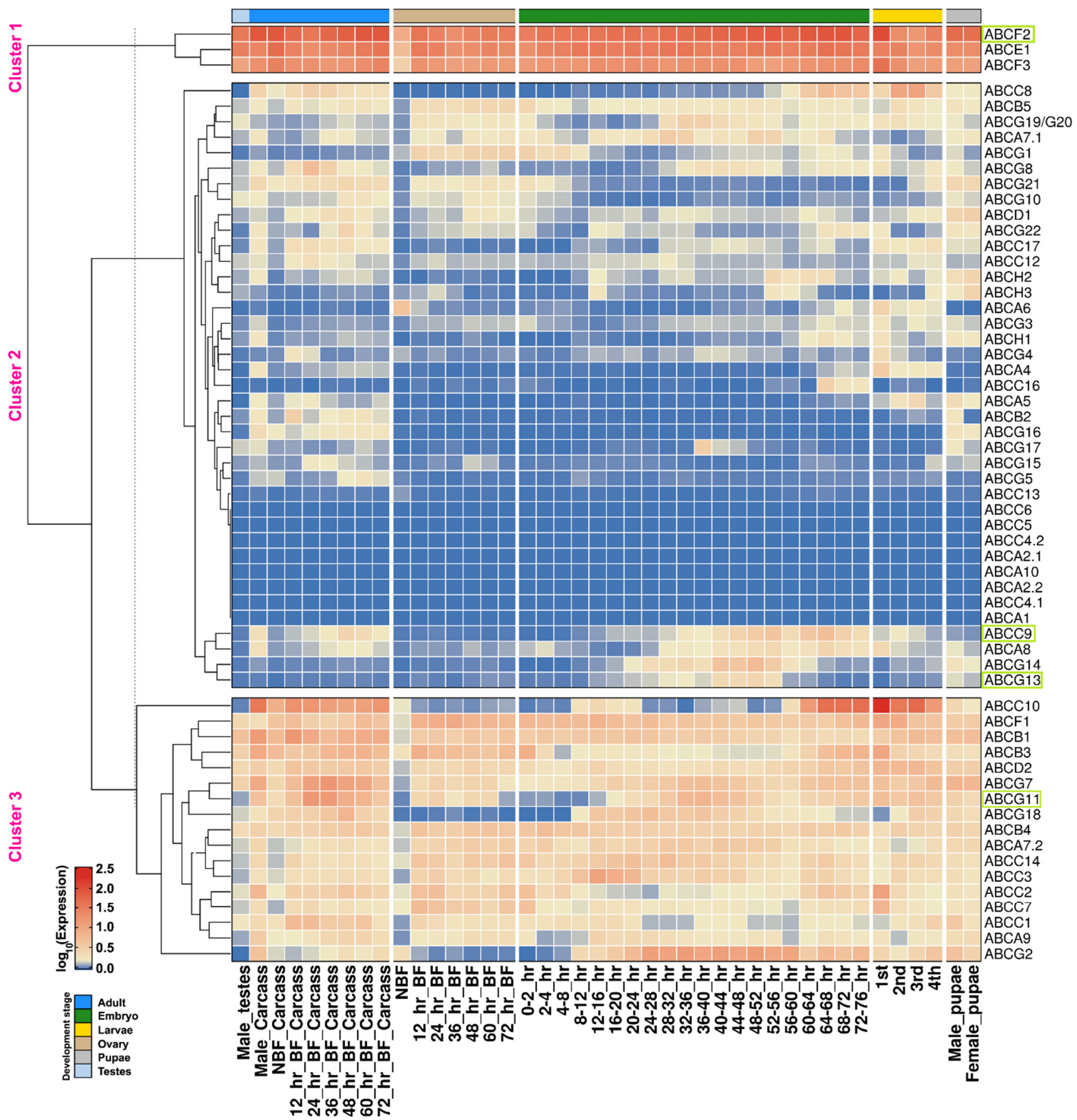

Figure 3. Expression profiling of Ae. aegypti ABC transporter transcripts in different developmental stages as well as at different time points after the blood-feeding using K-means clustering [45]. The Color scheme indicates the $\log _{10}$ FPKM expression value. NBF, non-blood-fed females; BF, blood-fed females; 1st to 4th instar stages of larvae. The expression pattern of few genes validated by $\mathrm{qPCR}$ is marked in boxes.

The genes that showed a decent to high-level expression throughout the mosquito development formed a separate cluster (Cluster 3, Figure 3). However, among these genes, AaeABCC10, AaeABCG2 and AaeABCG11 showed minimal expression in ovaries of blood-fed (BF) females. 


\subsection{Validating the Expression Pattern of Selected ABC Transporter Genes in Different Developmental Stages of Ae. aegypti}

To further validate the expression of randomly selected $A B C$ transporter genes (Aae$A B C C 9$, AaeABCF2, AaeABCG11 and AaeABCG13) in different developmental stages of mosquito i.e., eggs, larval stages, pupae and adult males or females, we performed qPCR (Figure 4) using gene-specific primers (primer sequences are given in Supplementary Table S1).
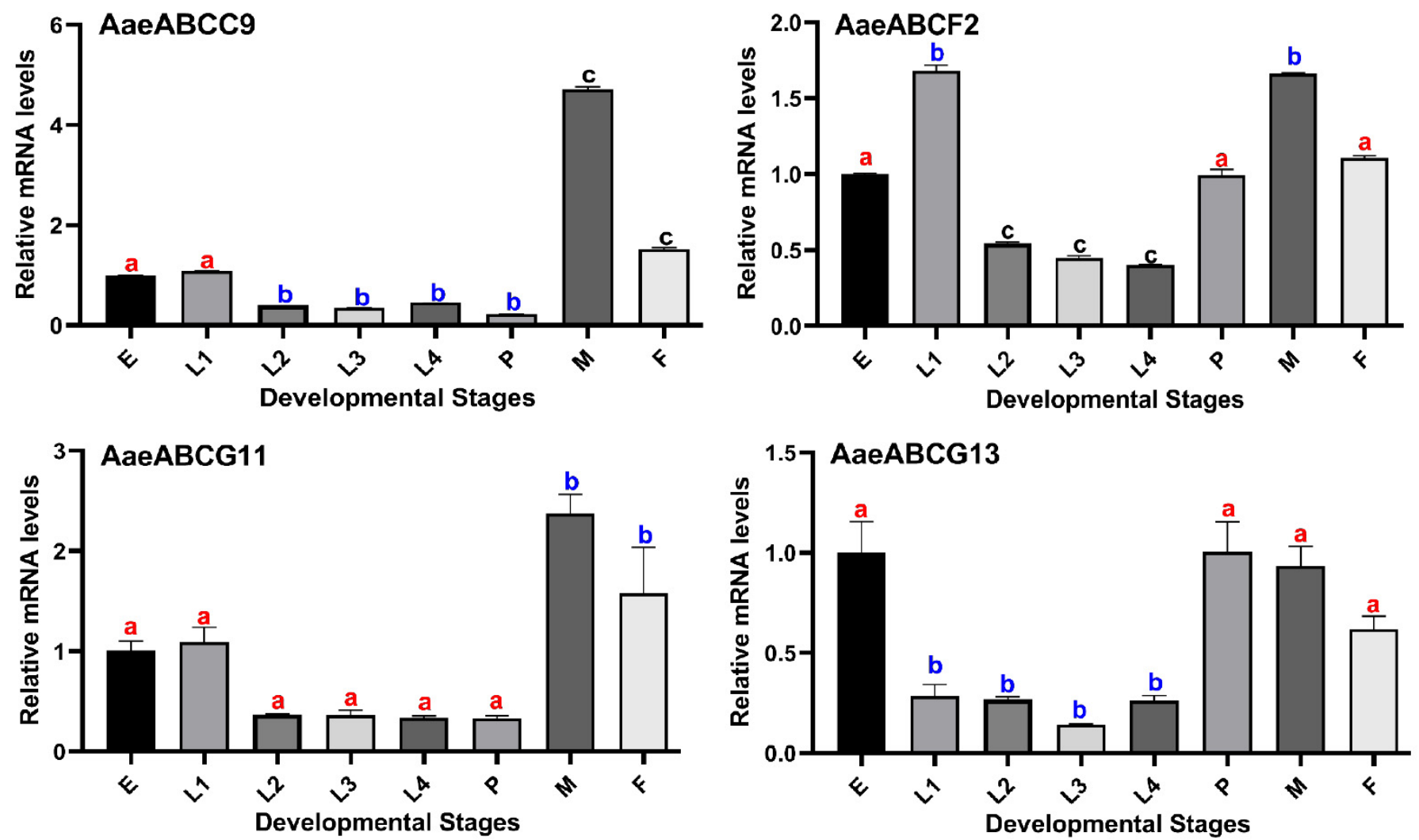

Figure 4. qPCR analysis of randomly selected $\mathrm{ABC}$ transporters in different developmental stages of Ae. aegypti mosquito. $\mathrm{E}=$ Eggs, $\mathrm{L}=$ Larvae (numbers indicate the instar of larvae L1 to L4), $\mathrm{P}=$ Pupae, $\mathrm{M}=$ Males and F = Females. All samples were collected from three biological replicates at each developmental stage, error bars indicate mean \pm SD $(n=3)$. The relative mRNA levels were calculated as mentioned in Materials and Methods. The mRNA levels in eggs were considered as 1.0 in each analysis. Significant differences among relative mRNA levels are represented with different alphabets like “a, b, c". The 'a' alphabet bearing bars have non-significant expression change to each other, while they have significant differences with bars bearing other alphabets $(b \& c)$.

The qPCR data of the selected genes (Figure 4) revealed a similar pattern when compared to the RNA-seq data (Figure 3). As mentioned previously, AaeABCC9 showed maximum expression in male carcass which is also observed in the qPCR data for this gene. AaeABCF2 gene shows expression throughout the development of mosquitoes with maximal expression in 1st instar larvae and adult stage (in both male and female) which is also evident in the qPCR data. AaeABCG13 expression was minimal during different developmental stages as per the RNA seq data and a similar pattern was observed after qPCR validation.

\subsection{Expression Profile of Ae. aegypti ABC Transporters upon Arboviral-Infections}

During arboviral infection in the mosquito, the virus must disseminate from the midgut (MG) through the body (CC) to the salivary gland (SG). Therefore, the gene expression is likely to be altered in different organs at various time points. To determine the spatial and sequential expression of Ae. aegypti $\mathrm{ABC}$ transporters, we analyzed datasets 
available from microarray experiments on three arboviral infections viz. DENV, YFV and WNV [7,46-48]. Infection kinetics was generated as per virus progression in the mosquito, beginning from its ingestion to readiness for subsequent transmission [on Day1, D2, D7, D10 and D14 post-infection (dpi)] in the whole mosquito as well as in its different organs including CC, MG and SG.

Most of the Ae. aegypti $\mathrm{ABC}$ transporters had a significant differential expression in the whole body of mosquito up to $7 \mathrm{dpi}$ (YFV, WNV and DENV). Our analyses detected the mRNA expression for 54 genes $(91.52 \%)$ in at least one of the arboviral infections at 1-7 dpi. Out of these 48 genes (81.35\%) showed differential expression in at least one of the arboviral infections (Figure 5). Forty-three genes were differentially expressed during YFV infection, 36 genes during WNV and 43 genes during DENV infection. Among these, 31 genes were commonly expressed during all three infections. AaeABCA7.2 and AaeABCC4.2 were exclusively expressed during the early infection of YFV while AaeABCA8, $A a e A B C B 3$ and $A a e A B C F 1$ were exclusively downregulated 7 dpi in DENV (Figure 5A). Almost all members of $A a e \mathrm{ABCB}, A a e \mathrm{ABCG}$ and $A a e \mathrm{ABCH}$ subfamilies showed continuous downregulation upon any of the three arboviral infections. In contrast few members of subfamilies AaeABCA (AaeABCA2.1, AaeABCA6), AaeABCC (AaeABCC7, AaeABCC10, AaeABCC14) and AaeABCF (AaeABCF2) were upregulated during arboviral infection. AaeABCC14 was continuously upregulated on all-time points of YFV, WNV and DENV infection [7].

Further to analyze the DENV infection kinetics we also investigated other available reports for $10 \mathrm{dpi}$ and $14 \mathrm{dpi}$ of DENV [46,47]. Contrary to the significantly differential expression of all $A B C$ transporters 1-7 dpi, only two genes from the ABCG subfamily were significantly modulated at 10 and $14 \mathrm{dpi}$, where AaeABCG3 gene was downregulated in the carcass at $10 \mathrm{dpi}$ of DENV [46] and AaeABCG11 was upregulated in the carcass and salivary gland on $14 \mathrm{dpi}$ of DENV infection [47]. Several ABC transporters were also found downregulated in the mosquito cell lines Aag2 upon DENV infection, similar to the in vivo experiments [48].

\subsection{Validating the Expression of ABC Transporter Genes in DENV2 Virus-Infected Ae. aegypti}

To validate the above transcriptome data (Figure 5), we randomly selected five $\mathrm{ABC}$ transporter genes (AaeABCC13, AaeABCE1, AaeABCF2, AaeABCG11 and AaeABCG13) and assessed their expression levels through qPCR. For the same, RNA was isolated from DENV2 infected Ae. aegypti as well as uninfected blood-fed mosquitoes at different time points. The actin gene was used as an internal control. The qPCR expression pattern of these genes revealed a similar trend as reported for the microarray data (Figure 6).

AaeABCC13 exhibited no differential regulation at early DENV2 infection (at 1 and $2 \mathrm{dpi}$ ) but was observed to be downregulated at $7 \mathrm{dpi}$, similar to the microarray reports. $A a e A B C E 1$ and $A a e A B C F 2$ transporters exhibited similar expression patterns post-DENV2 infection; both genes being significantly upregulated at the onset of infection ( $1 \mathrm{dpi})$, with subsequent downregulation as the infection progressed. In contrast, a continuous downregulation was observed for $A a e A B C G 11$ and AaeABCG13 genes post-DENV2 infection in accordance with the microarray data. 
A
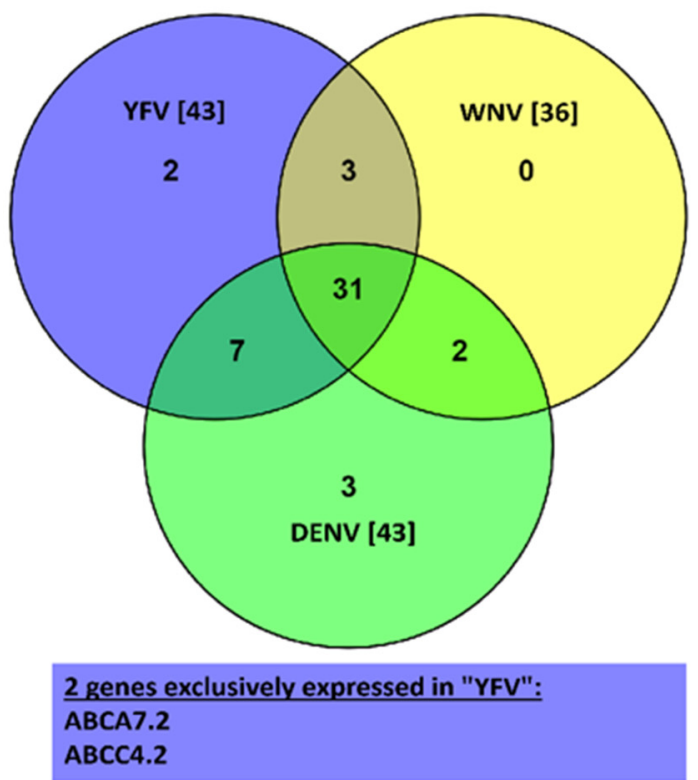

3 genes common to "YFV" and "WNV": ABCA1

ABCA10

ABCC8

2 genes common to "WNV" and "DENV":

AaeABCC3

AaeABCC7

3 genes exclusively expressed in "DENV":

ABCA8

ABCB3

ABCF1

7 genes common to "YFV" and "DENV":

AaeABCA9 AaeABCC17

AaeABCC4.1 AaeABCD1

AaeABCC12 AaeABCG13

AaeABCC13

31 genes commonly expressed in "YFV", "WNV" and "DENV":

$\begin{array}{llll}\text { ABCA2.1 } & \text { ABCC1 } & \text { ABCG3 } & \text { ABCH1 } \\ \text { ABCA4 } & \text { ABCC2 } & \text { ABCG8 } & \text { ABCH2 } \\ \text { ABCA5 } & \text { ABCC5 } & \text { ABCG10 } & \text { ABCH3 } \\ \text { ABCA5 } & \text { ABCC9 } & \text { ABCG11 } & \\ & \text { ABCC10 } & \text { ABCG14 } & \\ & \text { ABCC14 } & \text { ABCG15 } & \\ \text { ABCB1 } & & \text { ABCG16 } & \\ \text { ABCB2 } & & \text { ABCG17 } & \\ \text { ABCB4 } & \text { ABCD2 } & \text { ABCG18 } \\ \text { ABCB5 } & & \text { ABCG19/20 } \\ & \text { ABCF2 } & \text { ABCG21 } & \\ & & \text { ABCG22 } \\ & & & \end{array}$

B

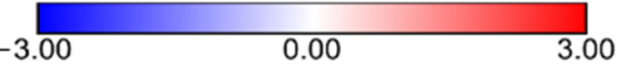

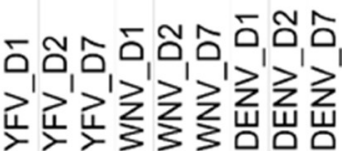

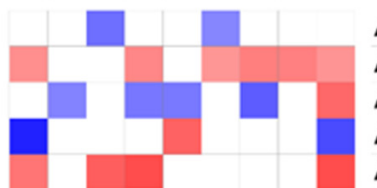

AaeABCA1

AaeABCA2.1

AaeABCA4

AaeABCA5

AaeABCA6

AaeABCA7.2

AaeABCA8

AaeABCA9

AaeABCA10

AaeABCB1

AaeABCB2

AaeABCB3

AaeABCB4

AaeABCB5

AaeABCC1

AaeABCC2

AaeABCC3

AaeABCC4.1

AaeABCC4.2

AaeABCC5

AaeABCC7

AaeABCC8

AaeABCC9

AaeABCC10

AaeABCC12

AaeABCC13

AaeABCC14

AaeABCC17

AaeABCD1

AaeABCD2

AaeABCF1

AaeABCF2

AaeABCG3

AaeABCG8

AaeABCG10

AaeABCG11

AaeABCG13

AaeABCG14

AaeABCG15

AaeABCG16

AaeABCG17

AaeABCG18

AaeABCG19/G20

AaeABCG21

AaeABCG22

AaeABCH1

$\mathrm{AaeABCH} 2$

$\mathrm{AaeABCH} 3$

Figure 5. Analysis of Ae. aegypti ABC transporters expression profile in WNV 2741, DENV-2 New Guinea C or YFV Asibi strain infected mosquitoes. As described by Colpitts et al., 2011, virus was used at 6.5 logs per mL, and $0.5 \mu \mathrm{L}$ was injected intra-thoracically per mosquito. The viral infection was confirmed by RT-qPCR [7]. (A) The Venn diagram represents the number of exclusively or commonly expressed genes post arboviral infection. The list of these genes is given at the bottom of the diagram. (B) Heatmap of log2 values of differentially regulated transcripts of Ae. aegypti ABC transporters at different days post virus infection [7]. Only the genes exhibiting a significant expression $(p<0.05)$ are represented here. The Color scheme indicates the log2 Fold-change expression of genes against controls at D1, D2, D7 (D = represents the day post-infection). 


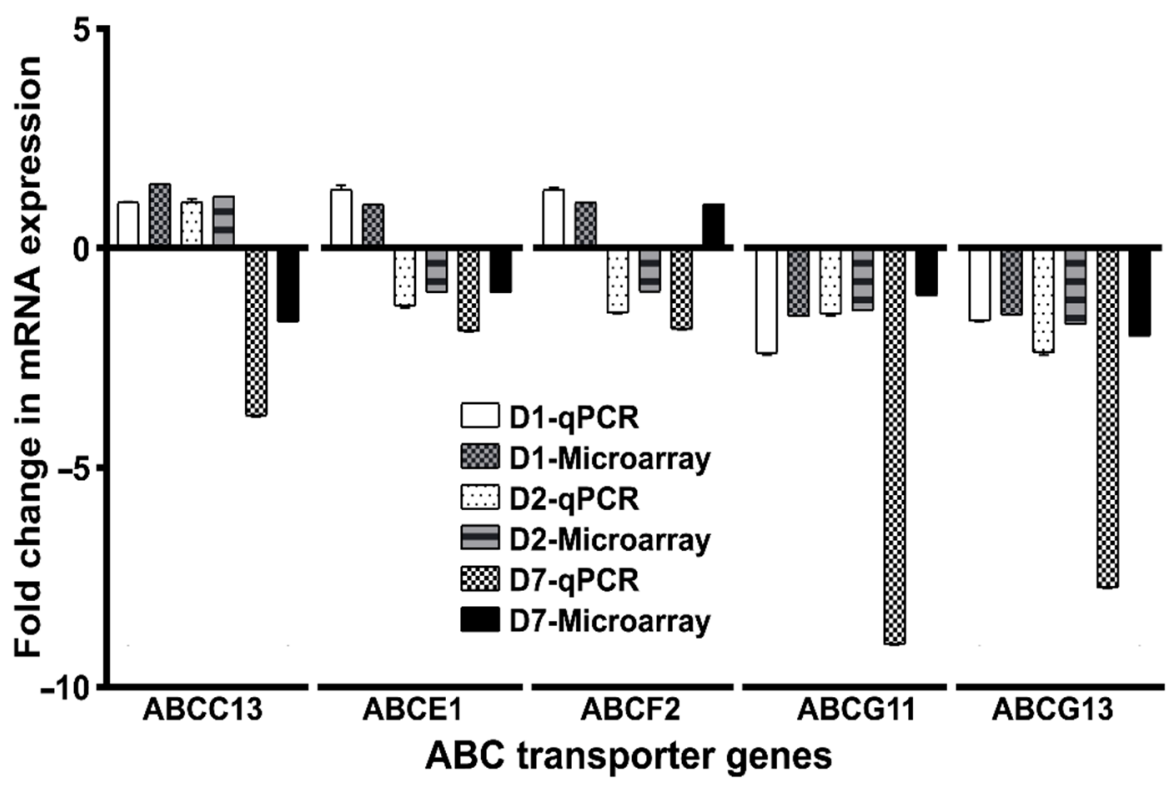

Figure 6. Comparative analysis of the microarray and qPCR expression data of a few representative $\mathrm{ABC}$ transporter genes (as mentioned on the x-axis) during DENV2 infection. The fold change in mRNA expression was calculated by ${ }^{\Delta \Delta} \mathrm{Ct}$ method as described in Materials and Methods, using the actin gene as an internal control. All samples were collected from three biological replicates of each treatment (DENV2 infected blood- v/s uninfected blood-fed) at specified time intervals. Error bars indicate mean $\pm \mathrm{SD}(n=3)$. D1, D2 and D7 indicate the days post-viral infection. The sequence of the primers used in qPCR is given in Supplementary Table S1.

\subsection{Analysis of Promoters and Transcription Factors Binding Site (TFBS) in The Regulatory Region of Virus-Induced ABC Transporters}

To understand the transcriptional regulation of $\mathrm{ABC}$ transporter genes having probable roles in mosquito immunity during arboviral infections, the binding sites for transcription factors (TFBS) were predicted in the $5^{\prime}$ UTR sequences of a few representative ABC transporter genes. We could identify the binding sites for STAT, Rel, Cnc, Bgb, Sch and Ets in these ABC transporter genes (Figure 7A), where the $5^{\prime}$ UTR sequences of each gene showed binding sites for single/multiple TFs. Further to correlate the possible transcriptional regulation of $\mathrm{ABC}$ transporter genes by these factors, we examined the expression patterns of these TFs in arboviral infected mosquito transcriptome [7]. Rel1 was observed to be upregulated in DENV infection with no significant change in WNV and YFV, however, Rel2 and Bgb showed downregulation in all arboviral infections. STAT was upregulated only at 2 dpi in DENV while Cnc variants showed upregulation at variable stages in all three arboviral infections. Interestingly, another transcription factor, Sch commonly associated with fatty-acid synthesis, was found to be upregulated throughout the three arboviral infections (till $7 \mathrm{dpi}$ ) (Figure 7B). The presence of immune mechanism related TFBS in $5^{\prime}$ UTR of these ABC transporter genes indicate their possible role during mosquito immunity. However, to identify the role of individual TF with specific ABC transporters, a time-dependent transcriptome profiling is required. 
A
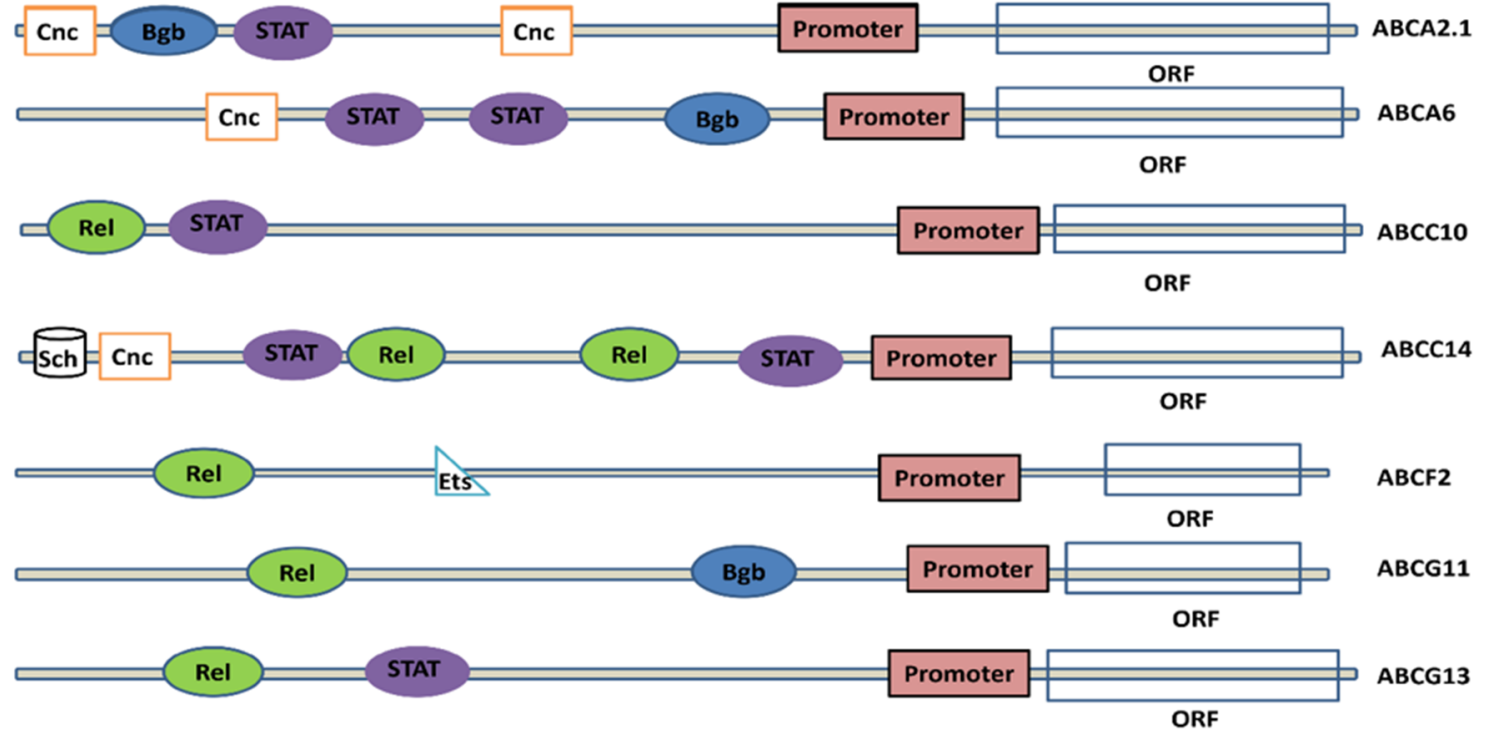

B
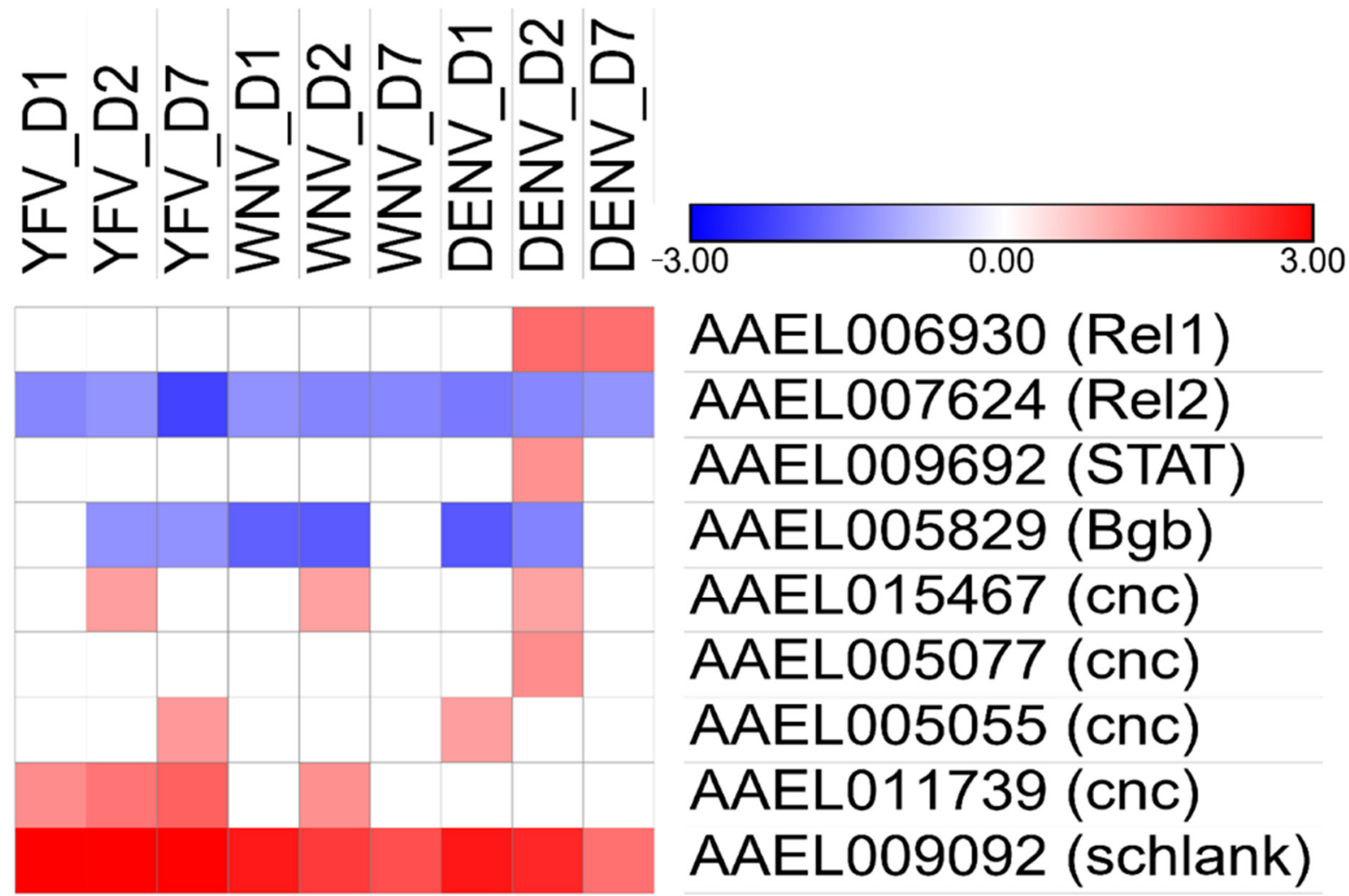

Figure 7. (A) Representation of putative transcription factors binding sites (TFBS) in the regulatory region of Ae. aegypti $\mathrm{ABC}$ transporter genes. ORF $=$ Open reading frames, STAT $=$ STAT binding site, Rel $=$ Rel binding site, Bgb $=$ Protein big brother binding site, $\mathrm{Cnc}=$ cap'n' collar binding site, Ets = Ets binding site and Sch = Schlank binding site. (B) Heatmap showing differential expression of transcription factor encoding genes in arboviral infected Ae. aegypti mosquitoes [7]. Only genes showing a significant $p$-value $(p<0.05)$ are shown here. D1, D2, D7 represent the days post arboviral infection.

\section{Discussion}

The involvement of $A B C$ transporters in multiple functions including lipid transport, multidrug resistance (MDR), transport of xenobiotics, chemotherapeutic drugs, and immunity, indicates their accessibility to a wide range of substrates and pathways $[49,50]$. However, the probable role of $A B C$ transporters in mosquito immune mechanisms postarboviral infections has not been elucidated. The identification of $A B C$ transporters in different mosquito genomes reveals interesting traits of these proteins in fundamental cellu- 
lar and housekeeping processes. Many of these proteins, even when belonging to the same subfamily often exhibit different functions, and proteins from different subfamilies may show related features. As detailed in earlier reports, and as observed in our phylogenetic analysis, the $\mathrm{ABC}$ transporters are highly conserved across various mosquito genus which are common hosts/vectors for different viruses including DENV, WNV, YFV, CHIKV etc. and protozoan parasites [7]. The conservation across these protein subfamilies in their functional domains may be attributed to their structural topology.

In the current study, the comparative sequence and phylogenetic analysis of 59 putative $A$ e. aegypti $\mathrm{ABC}$ transporters suggested a common evolutionary ancestral origin with other insect $\mathrm{ABC}$ transporters. There is a high level of conservation of Aae $\mathrm{ABC}$ transporters with orthologs from different insects, including the dipterans: secondary vector for DENV (Ae. albopictus), fruit fly (D. melanogaster), malaria vector mosquito (An. gambiae) and $C x$. quinquefasciatus, etc. The structural architecture of Aae $\mathrm{ABC}$ members is also highly conserved with their respective homologues from other insects, plants, and humans. Interestingly, the Aae $\mathrm{ABC}$ transporters subfamily E remains the smallest like other insects and humans; while, subfamilies $C$ and $\mathrm{G}$ have maximum members throughout insect genomes. Gene duplications was observed for AaeABCA2 (AaeABCA2.1 and AaeABCA2.2), AaeABCA7 (AaeABCA7.1 and AaeABCA7.2) and AaeABCC4 (AaeABCC4.1 and AaeABCC4.2) while there were certain gene deletions (AaeABCA3, AaeABCC11, AaeABCC15, and AaeABCG6) and gene additions (AaeABCA10, AaeABCC16, AaeABCC17, AaeABCG21 and AaeABCG22) when compared to An. gambiae transporters. The ABC transporters of Ae. aegypti still seems less in number when compared to $C x$. quinquefasciatus which has the highest number of $A B C$ transporters genes among the reported insect genomes; however, it has many pseudogenes and is still under curation. The Ae. aegypti $\mathrm{ABCB}$ subfamily members seem parallel with Anopheles and Drosophila having sequence homology of $\sim 30 \%$ with the human TAP proteins, which are involved in protein translocations for antigen processing [50]. As an extension to the defense mechanism, DmMdr65 (orthologue of AaeABCB2) has been reported to impart chemical protection to the insect brain and acts as the efflux transporter at the blood-brain barrier [51].

To ensure the functions of Ae. aegypti $\mathrm{ABC}$ transporters, we performed an in-depth comparative analyses of these genes through mosquito developmental stages and at different time-points post arboviral (YFV, WNV or DENV) infections. A few of the ABC transporters exhibited contrasting expression patterns during developmental stages and post arboviral infections, as observed for AaeABCA2.1 and AaeABCA6 genes. These transporters were upregulated during viral infections through $1 \mathrm{dpi}$ to $7 \mathrm{dpi}$, but their expression was scanty or minimal during mosquito development stages. AaeABCA4 was also seen upregulated at $7 \mathrm{dpi}$ on DENV infection while it had null expression during development stages $[7,45]$. This indicates the pro-viral role of these transporters. In contrast, Ae. aegypti $A B C A 8, A B C B 3$ and $A B C C 3$ genes were downregulated at $7 \mathrm{dpi}$, but they had relatively higher expression during development stages. Similarly, Ae. aegypti $A B C A 9, A B C B 2$, $A B C C 1, A B C C 9, A B C G 8, A B C G 10, A B C G 11, A B C G 13, A B C G 15$ to $A B C G 20$ transporters had optimum to high expression profile during development to adult stages, while they were mostly downregulated during arboviral infections [7,45]. Our analysis revealed commonly expressed genes in three arboviral infections suggesting a potentially conserved transcriptomic signature of arboviral infection regardless of the type of viral infection. To investigate the transcriptomic alteration of these transporters, we analyzed and compared the high throughput sequencing data with our qPCR expression profiles and found correlations in the expression of representative genes like Ae. aegypti ABCG11 and ABCG13. These genes showed high expression during adult stages of mosquito development similar to expression patterns observed during data analysis from Akbari et al. [45] Similarly, down-regulatory patterns were observed for these transporters during arboviral infection in both analyses (qPCR expression as well as bioinformatics analysis) [7]. These observations indicate the importance of these transporters during mosquito development while 
their downregulation on arboviral infection indicates the possible modulation of mosquito immune mechanisms by viruses.

The reported role of various $A B C$ transporters in different systems motivated us to further investigate their expression patterns. The downregulation of the peroxisomal $\mathrm{ABC}$ transporter $A a e A B C D 2$ on $2 \mathrm{dpi}$ and $7 \mathrm{dpi}$ of arbovirus suggested the modulation of peroxisomal machinery by the viruses for the establishment of infection in the host, as reported in the mammalian system [52]. Similarly, the downregulation of ABC transporters from subfamily $\mathrm{C}$ and $\mathrm{G}$ has been reported to play a role in the transportation of bacteriocin, glutathione conjugates and lipid-derived eicosanoids and is known to be involved in insect immune response to infection, indicating their role in antiviral immunity [18]. Further $A B C E 1$ role has been detailed during viral infection and anti-apoptosis in humans [39]. $A a e A B C E 1$ showed an increased expression during mosquito development and at $1 \mathrm{dpi}$ of DENV, however, it is downregulated as the infection progresses further, confirming its potential role in viral infection.

The arboviral infected Ae. aegypti salivary gland transcriptome analysis revealed upregulation of several ABC transporters from A, C, G and H (Supplementary Figure S2) suggesting their possible role in arboviral transmission [11,47]. Our analysis also predicted putative transcription factors for viral-induced Ae. aegypti ABC transporters, one of such is AaeABCC14, which is regulated by Rel, STAT, Cnc and Sch. Sch is involved in SREBP signaling, which is critical for viral propagation $[53,54]$ and AaeABCC14 is a putative sterol transporter. Both Sch and $A a e A B C C 14$ were upregulated in the analyzed transcriptomic profiles (Figures $5 \mathrm{~B}$ and $7 \mathrm{~B}$ ), indicating the possible regulatory involvement of Sch for AaeABCC14 gene.

These findings necessitate a detailed gene-specific study to establish the functions of above listed $\mathrm{ABC}$ transporters in mosquito immune development against arboviral infections. The results of this research will lay the foundation for functional research and genetic evolution analysis. The discovery of novel/non-canonical genes related to mosquito development and immunity is important for accelerating the transmissionblocking research to control mosquito-borne diseases.

\section{Materials and Methods}

\subsection{Ae. aegypti Mosquito Rearing}

Ae. aegypti mosquitoes were reared in a semi-natural insectarium at $27 \pm 1{ }^{\circ} \mathrm{C}, 80 \%$ relative humidity $(\mathrm{RH})$ and $12 \mathrm{~h}$ light:dark cycle as described before $[55,56]$. Larvae were fed on fish food (Gold Tokyo, Ahmedabad, India) while the adult mosquitoes were regularly fed on $10 \%$ sucrose solution ad libitum. For colony propagation, four to five-day-old females were fed on anesthetized mice and their eggs were collected in moist cups. The mice were maintained in the central animal facility and all the procedures were approved by the animal ethics committee (IAEC/RES/28/7).

\subsection{Identification and Classification of Ae. aegypti $A B C$ Transporters}

To identify putative $A e$. aegypti $\mathrm{ABC}$ transporters, protein sequences of already reported fifty-five An. gambiae ABC transporters [24,27] were retrieved from VectorBase [57] and subjected to BLASTp search against Ae. aegypti genome assembly 5 (AaegL5) [58]. The retrieved hits from $A e$. aegypti genome that exhibited blast e-value $<10^{-6}$ were further screened for $A B C$ transporter specific signature domains through the conserved domain database (CDD) tool at NCBI [59]. Furthermore, the profile hidden markov model was adopted to characterize the NBDs and TMDs in these identified putative Ae. aegypti ABC transporters [29].

Finally, the presence of $\mathrm{ABC}$ transporter sequences in Ae. aegypti was confirmed through BLASTp search in its genome using already identified ABC transporters from $D$. melanogaster and $C x$. quinquefasciatus as query. In addition, tBLASTn search was also performed using the protein sequences of An. gambiae $\mathrm{ABC}$ transporters to rule out the 
possibility of mis/un-annotated or new $\mathrm{ABC}$ transporter genes in Ae. aegypti genome assembly 5 (AaegL5).

The putative Ae. aegypti ABC transporters were classified into AaeABCA to AaeABCH subfamilies through multiple sequence alignment (Clustal Omega), phylogenetic tree reconstruction and orthologous relationship as reported before $[26,27,60]$. The nomenclature adopted for Ae. aegypti ABC transporters was based on their homology with An. gambiae $\mathrm{ABC}$ transporters [27]. The Ae. aegypti ABC transporters that exhibited one to one orthology with $A n$. gambiae ABC transporters were assigned the same nomenclature. However, when more than one Ae. aegypti ABC transporter exhibited orthology with a single ortholog in An. gambiae, the Ae. aegypti ABC transporter with maximum similarity was suffixed with X.1 while the one with less similarity was named X.2 and so on (where $X$ is the gene nomenclature corresponding to An. gambiae). Interestingly, some of the Ae. aegypti ABC transporters exhibited no orthology with $A n$. gambiae transporters and thus, they were named based on homology with their paralogs in Ae. aegypti.

\subsection{Phylogenetic Analysis of Ae. aegypti ABC Transporters}

To understand the evolutionary relationship of putative Ae. aegypti ABC transporters, we performed their phylogenetic analyses with An. gambiae, Cx. quinquefasciatus, $D$. melanogaster, and Ae. albopictus ABC transporters, using the neighbor-joining (NJ) method in MEGAX program [33]. The evolutionary distances were computed using the Poisson correction method and represented as the unit of number of amino acid substitutions per site. All positions with less than 95\% site coverage were eliminated, i.e., fewer than 5\% alignment gaps, missing data, and ambiguous bases were only allowed at any position (pairwise deletion). The tree topology confidence was assessed through bootstrap analysis using 1000 replicates.

\subsection{Transcriptomic Data Analysis of Ae. aegypti ABC Transporters}

To analyze the expression patterns of $\mathrm{ABC}$ transporters during mosquito development, all 42 RNA-seq libraries initially described by Akbari et al. [45] and already remapped to the Ae. aegypti AaegL5 assembly was retrieved from VectorBase (https: / vectorbase.org/ vectorbase/app/search/transcript/GenesByRNASeqEvidence\#GenesByRNASeqaaegLVP_ AGWG_SRP026319_ebi_rnaSeq_RSRC, accessed on 15 July 2020) and analyzed using R program. First, the raw counts per gene were calculated from RNA-seq data using "featureCounts" [60]. These raw counts were further converted to the fragments per kilobase per million mapped reads (FPKM) using "countToFPKM" module. The obtained FPKM values were log10-transformed (by adding 1 to it) before generating a heatmap by "ComplexHeatmap" using K-means clustering on rows as reported before [61]. Differential expression analysis was performed for selected groups using edgeR $[62,63]$.

Furthermore, to analyze the expression of Ae. aegypti ABC transporters during different arboviral infections like WNV, YFV or DENV, we constructed a single nonredundant gene list of these transporters based on prior AaegL1.2 and AaegL3.3 and current AaegL5.1 annotations. We analyzed the corresponding gene expression from the publicly available microarray datasets [7,46-48], wherein the reported probe sets were used to identify genes in new genome assembly through BLASTn. Genes identified with a significant $p$-value $<0.05$ were considered for expression analysis. The $\log _{2}$ fold change in the expression was used to construct a heatmap using Morpheus software (https:// software.broadinstitute.org/morpheus, latest accessed on 29 October 2020).

\subsection{Sample Collection and Analyzing the Expression Profile of Representative ABC Transporter} Genes Using $q P C R$

Different developmental stages of Ae. aegypti such as eggs, larvae (first to fourth-instar), pupae, adult males or females were collected in RNAlater and stored at $-80^{\circ} \mathrm{C}$. Total RNA was isolated from these samples using RNeasy mini kit (Qiagen, Hilden, Germany) with slight modification by adding $30 \mu \mathrm{L} \beta$-mercaptoethanol (2-ME) per $1 \mathrm{~mL}$ RLT buffer. First-strand cDNA was synthesized using QuantiTect reverse transcription kit (Qiagen, 
Hilden, Germany) according to manufacturer's instructions followed by qPCR using SYBR green supermix in an IQ5 multicolor detection system (Bio-Rad, Hercules, CA, USA), where the mRNA for mosquito ribosomal protein subunit $\mathrm{S} 6$ gene was used as a normalization control [56]. The primer sets of representative genes used for expression analyses are mentioned in supplementary Table S1. Following PCR cycle parameters were used, initial denaturation at $95{ }^{\circ} \mathrm{C}$ for $3 \mathrm{~min}, 35$ cycles of $10 \mathrm{~s}$ at $95{ }^{\circ} \mathrm{C}, 40 \mathrm{~s}$ at $55^{\circ} \mathrm{C}$, and $1 \mathrm{~min}$ at $72{ }^{\circ} \mathrm{C}$. The fluorescence readings were taken after each cycle. A final extension at $72{ }^{\circ} \mathrm{C}$ for $10 \mathrm{~min}$ was performed followed by a melting curve analysis.

\subsection{Viral Infection, Sample Collection and Expression Analysis of Representative ABC Transporter Genes}

Three-to-five-day old female mosquitoes were starved overnight and offered an infectious blood meal containing rabbit blood washed with $1 \times$ PBS and supplemented with $10 \mathrm{mM}$ ATP (Thermo Scientific, Waltham, MA, USA), 10\% fetal bovine serum (Sigma, Kawasaki, Japan), 7.5\% Sodium bicarbonate and DENV2 virus titer suspended in RPMI media (Gibco, Scotland, United Kingdom) maintained at $1 \times 10^{6} \mathrm{pfu} / \mathrm{mL}$. Control mosquitoes were fed with the same blood meal composition except for the virus. Fully engorged females were selected and kept in a cage with $10 \%$ sucrose solution ad libitum in incubation at standard rearing conditions. Sham treated control and infected mosquitoes were collected at 1,2 and 7 dpi.

Total RNA was extracted from the collected samples using E.Z.N.A. Total RNA kit I (Omega Bio-tek, Norcross, GA, USA) and isolated RNA was treated with DNase using Turbo DNA-free kit (Thermo Fisher Scientific), and reverse transcribed by iScript cDNA synthesis kit (Biorad, Hercules, CA, USA). Gene expression was quantified in LightCycler 96 qPCR system (Roche, Mannheim, Germany) using EvaGreen Master Mix (Euromedex, Lyon, France) following standard protocol, using gene-specific primers as detailed in supplementary Table S1. Mosquito actin gene expression was used as a normalization control [64]. The reactions were performed using the following cycle conditions: an initial $95{ }^{\circ} \mathrm{C}$ for $5 \mathrm{~min}, 40$ cycles of $95^{\circ} \mathrm{C}$ for $15 \mathrm{~s}, 60^{\circ} \mathrm{C}$ for $20 \mathrm{~s}$ and $72{ }^{\circ} \mathrm{C}$ for $20 \mathrm{~s}$ followed by a melting curve analysis. Fold changes were calculated using the ${ }^{\Delta \Delta} \mathrm{Ct}$ method as described before [65].

All the data were expressed as mean \pm standard deviation (SD). Statistical significance was analyzed by one-way ANOVA with Tukey's multiple comparison test for the differential gene expression using GraphPad Prism 8.0 (GraphPad Software, La Jolla, CA, USA). The data with a $p$-value $<0.05$ were considered significant.

\subsection{Identification of Promoter and Transcription Factor Binding Sites in ABC Transporter Genes}

To identify promoters, the $5^{\prime}$ upstream sequence of each Ae. aegypti ABC transporter gene was analyzed using the online NPP2.2 tool (http://www.fruitfly.org/seq_tools / promoter.html, accessed on 29 March 2021), (organism selected = Eukaryotes and Minimum promoter score $=0.8$ ) [66]. The upstream $5^{\prime} \mathrm{UTR}$ sequence to promoter of selected ABC transporter genes was analyzed with online JASPAR software (http:/ /jaspar.genereg.net/ search?q=\&collection=CORE\&tax_group=insects, accessed on 31 March 2021) to predict the transcription factor binding (TFB) sites. Here the insect core database was selected, using Drosophila as a reference organism and a similarity threshold of $80 \%$ [67].

Supplementary Materials: The following are available online at https: / www.mdpi.com/article / 10.3390/pathogens10091127/s1, Supplementary Figure S1: (A-E) Subfamily wise phylogeny of Ae. aegypti (AAEL) ABC transporters with its homologs from An. gambiae (AGAP), Ae. albopictus (AALF), Cx. quinquefasciatus (CPIJ) and D. melanogaster (Dmel). Each gene in the phylogeny is designated in the format: species acronym followed by Gene Id and subfamily member name. The Gene Id for mosquito genes were retrieved from VectorBase and for Drosophila genes from Roth et al., 2003 [24]. The phylogenetic tree was constructed using the neighbor-joining method as described in materials and methods. The bootstrap value is calculated from 1000 replicates and marked on each corresponding node. Supplementary Figure S1A: Phylogenetic tree of Ae. aegypti (AAEL) ABC 
transporter subfamily A (ABCA) with its homologs from other insects. Supplementary Figure S1B: Phylogenetic tree of Ae. aegypti (AAEL) ABC transporter subfamily B (ABCB) with its homologs from other insects. Supplementary Figure S1C: Phylogenetic tree of Ae. aegypti (AAEL) ABC transporter subfamily $C$ (ABCC) with its homologs from other insects. Supplementary Figure S1D: Phylogenetic tree of Ae. aegypti (AAEL) ABC transporter subfamilies D-F and H (ABCD, ABCE, ABCF and ABCH) with their respective homologs from other insects. Supplementary Figure S1E: Phylogenetic tree of Ae. aegypti (AAEL) ABC transporter subfamily G (ABCG) with its homologs from other insects. Supplementary Figure S2. Expression profiles of Ae. aegypti ABC transporters post arboviral (ZIKV, CHIKV or DENV2) infection in mosquito salivary glands [11]. The Colour scheme indicates the $\log _{2}$ Fold-change expression of genes mentioned and D7 and D14 represent the days post-infection. Supplementary Table S1: List of primers used for qPCR to validate the ABC transporter genes expression during mosquito development and post-arboviral infection (as mentioned in materials and methods Sections 4.5 and 4.6). Supplementary Table S2: List of immunity related transcription factors identified in the $5^{\prime}$ UTR sequences of representative ABC transporters and their functions [67]. Supplementary File Excel 1: Supplementary File contains various supporting details for this manuscript as follows- sheet 1- Identifiers for Ae. aegypti ABC transporter genes used in current and previous studies under sheet name "1AaeABCs Comparison oldvsnew" [26,28]. Sheet 2-Ae. aegypti ABC transporters expression data used to construct heatmap (Figure 3 in main manuscript), based on RNA-seq libraries from Akbari et al., 2013 under sheet name "2AaeABCsDevelopmentalexpression" [45]. Sheet 3-Microarray Expression data of Ae. aegypti ABC transporters post arboviral (YFV, WNV or DENV) infection to mosquito (Colpitts et al., 2011) (Figure 5 in main manuscript) under sheet name "3AaeABCs Immune expression" [7].

Author Contributions: Conceptualization, V.K., S.G., L.G., S.K. and V.S.; methodology, V.K., S.K. and V.S.; formal analysis and data curation, V.K. and V.S.; Performed the experiments V.K. and C.T.D. (V.K. also performed the experiments at the University of Montpellier under the EMBO short term fellowship); writing-original draft preparation, V.K., S.K. and V.S.; writing-review and editing, V.K., S.G., L.G., K.G., D.M., J.P., S.K. and V.S.; visualization, V.K.; supervision, V.S., S.K., S.G. and J.P.; funding acquisition, V.K., L.G. and S.K. All authors have read and agreed to the published version of the manuscript.

Funding: This research was funded by the Department of Biotechnology (DBT), Government of India (Grant No. BT/PR8758/AGR/36/744/2013) awarded to S.K. and L.G., V.K. received Senior Research fellowship (F/41/2018-ECD-II) from Indian Council of Medical Research (ICMR), Government of India and EMBO Short-term fellowship (EMBO STF_8336) from European Molecular Biology Organization, Germany.

Institutional Review Board Statement: The study was conducted according to the guidelines of the Declaration of Helsinki, and approved by the Ethics Committee of Institute Biosafety Committee (protocol code BITS/IBSC/ 2018/02, approval date: 10 March 2018 (2018-2021); Institute Animal Ethics Committee (protocol code IAEC/RES/23/18, approval date: 28 October 2017 (2017-19)) and (protocol code IAEC/RES/28/7, approval date: 10 October 2020 (2020-22)).

Informed Consent Statement: Not applicable.

Data Availability Statement: The data presented in this study are available in supplementary materials and it was taken from existed microarray and RNA-seq datasets under accessions: GSE28208, SRR8921123-8921132, SRP026319 [7,11,45].

Acknowledgments: V.K., S.G., L.G., K.G., S.K. and V.S. wish to acknowledge BITS Pilani for the infrastructural support. L.G. and S.K. also wish to acknowledge CBLU, Bhiwani, Haryana. All authors acknowledge the helping staff from BITS, Pilani and MIVEGEC, Univ. Montpellier, IRD, CNRS, France.

Conflicts of Interest: The authors declare no conflict of interest.

\section{References}

1. Bhatt, S.; Gething, P.W.; Brady, O.J.; Messina, J.P.; Farlow, A.W.; Moyes, C.L.; Drake, J.M.; Brownstein, J.S.; Hoen, A.G.; Sankoh, O.; et al. The global distribution and burden of dengue. Nature 2013, 496, 504-507. [CrossRef] [PubMed]

2. Kraemer, M.U.G.; Reiner, R.C.; Brady, O.J.; Messina, J.P.; Gilbert, M.; Pigott, D.M.; Yi, D.; Johnson, K.; Earl, L.; Marczak, L.B.; et al. Past and future spread of the arbovirus vectors Aedes aegypti and Aedes albopictus. Nat. Microbiol. 2019, 4, 854-863. [CrossRef] 
3. Fauci, A.S.; Morens, D.M. Zika virus in the americas-yet another arbovirus threat. N. Engl. J. Med. 2016, 374, 601-604. [CrossRef] [PubMed]

4. Wu, P.; Yu, X.; Wang, P.; Cheng, G. Arbovirus lifecycle in mosquito: Acquisition, propagation and transmission. Expert Rev. Mol. Med. 2019, 21, e1. [CrossRef] [PubMed]

5. Tchankouo-Nguetcheu, S.; Khun, H.; Pincet, L.; Roux, P.; Bahut, M.; Huerre, M.; Guette, C.; Choumet, V. Differential protein modulation in midguts of Aedes aegypti infected with chikungunya and dengue 2 viruses. PLoS ONE 2010, 5, e13149. [CrossRef]

6. Luplertlop, N.; Surasombatpattana, P.; Patramool, D.; Dumas, E.; Wasinpiyamongkol, L.; Saune, L.; Hamel, R.; Bernard, E.; Sereno, D.; Thomas, F.R.; et al. Induction of a peptide with activity against a broad spectrum of pathogens in the Aedes aegypti salivary gland, following infection with Dengue Virus. PLoS Pathog. 2011, 7, e1001252. [CrossRef] [PubMed]

7. Colpitts, T.M.; Cox, J.; Vanlandingham, D.L.; Feitosa, F.M.; Cheng, G.; Kurscheid, S.; Wang, P.; Krishnan, M.N.; Higgs, S.; Fikrig, E. Alterations in the aedes aegypti transcriptome during infection with west nile, dengue and yellow fever viruses. PLoS Pathog. 2011, 7, e1002189. [CrossRef]

8. Carvalho-Leandro, D.; Ayres, C.F.J.; Guedes, D.R.D.; Suesdek, L.; Melo-Santos, M.A.V.; Oliveira, C.F.; Cordeiro, M.T.; Regis, L.N.; Marques, E.T.; Gil, L.H.; et al. Immune transcript variations among Aedes aegypti populations with distinct susceptibility to dengue virus serotype 2. Acta Trop. 2012, 124, 113-119. [CrossRef]

9. Kingsolver, M.B.; Huang, Z.; Hardy, R.W. Insect antiviral innate immunity: Pathways, effectors, and connections. J. Mol. Biol. 2013, 425, 4921-4936. [CrossRef] [PubMed]

10. Souza-Neto, J.A.; Sim, S.; Dimopoulos, G. An evolutionary conserved function of the JAK-STAT pathway in anti-dengue defense. Proc. Natl. Acad. Sci. USA 2009, 106, 17841-17846. [CrossRef]

11. Chowdhury, A.; Modahl, C.M.; Tan, S.T.; Wei Xiang, B.W.; Missé, D.; Vial, T.; Kini, R.M.; Pompon, J.F. JNK pathway restricts DENV2, ZIKV and CHIKV infection by activating complement and apoptosis in mosquito salivary glands. PLoS Pathog. 2020, 16, e1008754. [CrossRef]

12. Khoo, C.C.H.; Doty, J.B.; Heersink, M.S.; Olson, K.E.; Franz, A.W.E. Transgene-mediated suppression of the RNA interference pathway in Aedes aegypti interferes with gene silencing and enhances Sindbis virus and dengue virus type 2 replication. Insect Mol. Biol. 2013, 22, 104-114. [CrossRef]

13. Sim, S.; Dimopoulos, G. Dengue virus inhibits immune responses in Aedes aegypti cells. PLoS ONE 2010, 5, e10678. [CrossRef] [PubMed]

14. Sigle, L.T.; McGraw, E.A. Expanding the canon: Non-classical mosquito genes at the interface of arboviral infection. Insect Biochem. Mol. Biol. 2019, 109, 72-80. [CrossRef]

15. Croker, B.; Crozat, K.; Berger, M.; Xia, Y.; Sovath, S.; Schaffer, L.; Eleftherianos, I.; Imler, J.L.; Beutler, B. ATP-sensitive potassium channels mediate survival during infection in mammals and insects. Nat. Genet. 2007, 39, 1453-1460. [CrossRef] [PubMed]

16. Figueira-Mansur, J.; Ferreira-Pereira, A.; Mansur, J.F.; Franco, T.A.; Alvarenga, E.S.L.; Sorgine, M.H.F.; Neves, B.C.; Melo, A.C.A.; Leal, W.S.; Masuda, H.; et al. Silencing of P-glycoprotein increases mortality in temephos-treated Aedes aegypti larvae. Insect Mol. Biol. 2013, 22, 648-658. [CrossRef] [PubMed]

17. Félix, R.C.; Müller, P.; Ribeiro, V.; Ranson, H.; Silveira, H. Plasmodium infection alters Anopheles gambiae detoxification gene expression. BMC Genom. 2010, 11, 312. [CrossRef]

18. Stanley, D.; Kim, Y. Prostaglandins and other eicosanoids in insects: Biosynthesis and biological actions. Front. Physiol. 2019, 9, 1927. [CrossRef]

19. Rees, D.C.; Johnson, E.; Lewinson, O. ABC transporters: The power to change. Nat. Rev. Mol. Cell Biol. 2009, 10, 218-227. [CrossRef]

20. Linton, K.J. Structure and function of ABC transporters. Physiology 2007, 22, 122-130. [CrossRef]

21. Wilkens, S. Structure and mechanism of ABC transporters. F1000Prime Rep. 2015, 7. [CrossRef]

22. Dermauw, W.; Van Leeuwen, T. The ABC gene family in arthropods: Comparative genomics and role ininsecticide transport and resistance. Insect Biochem. Mol. Biol. 2014, 45, 89-110. [CrossRef]

23. Hollenstein, K.; Dawson, R.J.; Locher, K.P. Structure and mechanism of ABC transporter proteins. Curr. Opin. Struct. Biol. 2007, 17, 412-418. [CrossRef]

24. Roth, C.W.; Holm, I.; Graille, M.; Dehoux, P.; Rzhetsky, A.; Wincker, P.; Weissenbach, J.; Brey, P.T. Identification of the Anopheles gambiae ATP-binding cassette transporter superfamily genes. Mol. Cells 2003, 15, 150-158.

25. Matthews, B.J.; Dudchenko, O.; Kingan, S.B.; Koren, S.; Antoshechkin, I.; Crawford, J.E.; Glassford, W.J.; Herre, M.; Redmond, S.N.; Rose, N.H.; et al. Improved reference genome of Aedes aegypti informs arbovirus vector control. Nature 2018, 563, 501-507. [CrossRef]

26. Lu, H.; Xu, Y.; Cui, F. Phylogenetic analysis of the ATP-binding cassette transporter family in three mosquito species. Pestic. Biochem. Physiol. 2016, 132, 118-124. [CrossRef] [PubMed]

27. Pignatelli, P.; Ingham, V.A.; Balabanidou, V.; Vontas, J.; Lycett, G.; Ranson, H. The Anopheles gambiae ATP-binding cassette transporter family: Phylogenetic analysis and tissue localization provide clues on function and role in insecticide resistance. Insect Mol. Biol. 2018, 27, 110-122. [CrossRef] [PubMed]

28. Figueira-Mansur, J.; Schrago, C.G.; Salles, T.S.; Alvarenga, E.S.L.; Vasconcellos, B.M.; Melo, A.C.A.; Moreira, M.F. Phylogenetic analysis of the ATP-binding cassette proteins suggests a new ABC protein subfamily J in Aedes aegypti (Diptera: Culicidae). BMC Genom. 2020, 21, 463. [CrossRef] 
29. Potter, S.C.; Luciani, A.; Eddy, S.R.; Park, Y.; Lopez, R.; Finn, R.D. HMMER web server: 2018 update. Nucleic Acids Res. 2018, 46, W200-W204. [CrossRef] [PubMed]

30. Liu, S.; Zhou, S.; Tian, L.; Guo, E.; Luan, Y.; Zhang, J.; Li, S. Genome-wide identification and characterization of ATP-binding cassette transporters in the silkworm, Bombyx mori. BMC Genom. 2011, 12, 491. [CrossRef] [PubMed]

31. Broehan, G.; Kroeger, T.; Lorenzen, M.; Merzendorfer, H. Functional analysis of the ATP-binding cassette (ABC) transporter gene family of Tribolium castaneum. BMC Genom. 2013, 14, 6. [CrossRef]

32. He, Q.; Yan, Z.; Si, F.; Zhou, Y.; Fu, W.; Chen, B. ATP-binding cassette (ABC) transporter genes involved in pyrethroid resistance in the malaria vector Anopheles sinensis: Genome-wide identification, characteristics, phylogenetics, and expression profile. Int. J. Mol. Sci. 2019, 20, 1409. [CrossRef]

33. Kumar, S.; Stecher, G.; Li, M.; Knyaz, C.; Tamura, K. MEGA X: Molecular evolutionary genetics analysis across computing platforms. Mol. Biol. Evol. 2018, 35, 1547. [CrossRef] [PubMed]

34. Dean, M.; Rzhetsky, A.; Allikmets, R. The human ATP-binding cassette (ABC) transporter superfamily. Genome Res. 2001, 42, 1007-1017. [CrossRef]

35. Bariami, V.; Jones, C.M.; Poupardin, R.; Vontas, J.; Ranson, H. Gene amplification, abc transporters and cytochrome p450s: Unraveling the molecular basis of pyrethroid resistance in the dengue vector, aedes aegypti. PLoS Negl. Trop. Dis. 2012, 6, e1692. [CrossRef] [PubMed]

36. Buss, D.S.; Callaghan, A. Interaction of pesticides with p-glycoprotein and other ABC proteins: A survey of the possible importance to insecticide, herbicide and fungicide resistance. Pestic. Biochem. Physiol. 2008, 90, 141-153. [CrossRef]

37. Theodoulou, F.L.; Holdsworth, M.; Baker, A. Peroxisomal ABC transporters. FEBS Lett. 2006, 580, 1139-1155. [CrossRef] [PubMed]

38. Paytubi, S.; Wang, X.; Lam, Y.W.; Izquierdo, L.; Hunter, M.J.; Jan, E.; Hundal, H.S.; Proud, C.G. ABC50 promotes translation initiation in mammalian cells. J. Biol. Chem. 2009, 284, 24061-24073. [CrossRef] [PubMed]

39. Tian, Y.; Han, X.; Tian, D.L. The biological regulation of ABCE1. IUBMB Life 2012, 64, 795-800. [CrossRef]

40. Melby, T.E.; Ciampaglio, C.N.; Briscoe, G.; Erickson, H.P. The symmetrical structure of structural maintenance of chromosomes (SMC) and MukB proteins: Long, antiparallel coiled coils, folded at a flexible hinge. J. Cell Biol. 1998, 142, 1595-1604. [CrossRef] [PubMed]

41. Soppa, J. Prokaryotic structural maintenance of chromosomes (SMC) proteins: Distribution, phylogeny, and comparison with MukBs and additional prokaryotic and eukaryotic coiled-coil proteins. Gene 2001, 278, 253-264. [CrossRef]

42. Kerr, I.D. Structure and association of ATP-binding cassette transporter nucleotide-binding domains. Biochim. Biophys. Acta Biomembr. 2002, 1561, 47-64. [CrossRef]

43. Sánchez-Fernández, R.; Davies, T.G.E.; Coleman, J.O.D.; Rea, P.A. The Arabidopsis thaliana ABC Protein Superfamily, a Complete Inventory. J. Biol. Chem. 2001, 276, 30231-30244. [CrossRef] [PubMed]

44. Thomas, C.; Tampé, R. Structural and Mechanistic Principles of ABC Transporters. Annu. Rev. Biochem. 2020, 89, 605-636. [CrossRef]

45. Akbari, O.S.; Antoshechkin, I.; Amrhein, H.; Williams, B.; Diloreto, R.; Sandler, J.; Hay, B.A. The developmental transcriptome of the mosquito Aedes aegypti, an invasive species and major arbovirus vector. G3 Genes Genomes Genet. 2013, 3, 1493-1509. [CrossRef]

46. Xi, Z.; Ramirez, J.L.; Dimopoulos, G. The Aedes aegypti toll pathway controls dengue virus infection. PLoS Pathog. 2008, 4, e1000098. [CrossRef]

47. Sim, S.; Ramirez, J.L.; Dimopoulos, G. Dengue virus infection of the aedes aegypti salivary gland and chemosensory apparatus induces genes that modulate infection and blood-feeding behavior. PLoS Pathog. 2012, 8, e1002631. [CrossRef] [PubMed]

48. Barletta, A.B.F.; Alves, L.R.; Nascimento Silva, M.C.L.; Sim, S.; Dimopoulos, G.; Liechocki, S.; Maya-Monteiro, C.M.; Sorgine, M.H.F. Emerging role of lipid droplets in Aedes aegypti immune response against bacteria and Dengue virus. Sci. Rep. 2016, 6, 19928. [CrossRef] [PubMed]

49. Labbé, R.; Caveney, S.; Donly, C. Genetic analysis of the xenobiotic resistance-associated ABC gene subfamilies of the Lepidoptera. Insect Mol. Biol. 2011, 20, 243-256. [CrossRef]

50. Abele, R.; Tampé, R. The ABCs of immunology: Structure and function of TAP, the transporter associated with antigen processing. Physiology 2004, 19, 216-224. [CrossRef] [PubMed]

51. Mayer, F.; Mayer, N.; Chinn, L.; Pinsonneault, R.L.; Kroetz, D.; Bainton, R.J. Evolutionary conservation of vertebrate blood-brain barrier chemoprotective mechanisms in drosophila. J. Neurosci. 2009, 29, 3538-3550. [CrossRef] [PubMed]

52. You, J.; Hou, S.; Malik-Soni, N.; Xu, Z.; Kumar, A.; Rachubinski, R.A.; Frappier, L.; Hobman, T.C. Flavivirus Infection Impairs Peroxisome Biogenesis and Early Antiviral Signaling. J. Virol. 2015, 89, 12349-12361. [CrossRef] [PubMed]

53. Fu, Q.; Inankur, B.; Yin, J.; Striker, R.; Lan, Q. Sterol Carrier Protein 2, a Critical Host Factor for Dengue Virus Infection, Alters the Cholesterol Distribution in Mosquito Aag2 Cells. J. Med. Entomol. 2015, 52, 1124-1134. [CrossRef] [PubMed]

54. Cloherty, A.P.M.; Olmstead, A.D.; Ribeiro, C.M.S.; Jean, F. Hijacking of lipid droplets by hepatitis C, dengue and zika viruses-from viral protein moonlighting to extracellular release. Int. J. Mol. Sci. 2020, 21, 7901. [CrossRef] [PubMed]

55. Kajla, M.; Bhattacharya, K.; Gupta, K.; Banerjee, U.; Kakani, P.; Gupta, L.; Kumar, S. Identification of the Temperature Induced Larvicidal Efficacy of Agave angustifolia against Aedes, Culex, and Anopheles Larvae. Front. Public Health 2016, 3, 286. [CrossRef] [PubMed] 
56. Molina-Cruz, A.; Gupta, L.; Richardson, J.; Bennett, K.; Black IV, W.; Barillas-Mury, C. Effect of mosquito midgut trypsin activity on dengue-2 virus infection and dissemination in Aedes aegypti. Am. J. Trop. Med. Hyg. 2005, 72, 631-637. [CrossRef]

57. Giraldo-Calderón, G.I.; Emrich, S.J.; MacCallum, R.M.; Maslen, G.; Emrich, S.; Collins, F.; Dialynas, E.; Topalis, P.; Ho, N.; Gesing, S.; et al. VectorBase: An updated Bioinformatics Resource for invertebrate vectors and other organisms related with human diseases. Nucleic Acids Res. 2015, 43, D707-D713. [CrossRef]

58. Altschul, S.F.; Gish, W.; Miller, W.; Myers, E.W.; Lipman, D.J. Basic local alignment search tool. J. Mol. Biol. 1990, 215, 403-410. [CrossRef]

59. Marchler-Bauer, A.; Bryant, S.H. CD-Search: Protein domain annotations on the fly. Nucleic Acids Res. 2004, 32, W327-W331. [CrossRef]

60. Liao, Y.; Smyth, G.K.; Shi, W. FeatureCounts: An efficient general purpose program for assigning sequence reads to genomic features. Bioinformatics 2014, 30, 923-930. [CrossRef]

61. Gu, Z.; Eils, R.; Schlesner, M. Complex heatmaps reveal patterns and correlations in multidimensional genomic data. Bioinformatics 2016, 32, 2847-2849. [CrossRef] [PubMed]

62. Robinson, M.D.; McCarthy, D.J.; Smyth, G.K. edgeR: A Bioconductor package for differential expression analysis of digital gene expression data. Bioinformatics 2009, 26, 139-140. [CrossRef]

63. McCarthy, D.J.; Chen, Y.; Smyth, G.K. Differential expression analysis of multifactor RNA-Seq experiments with respect to biological variation. Nucleic Acids Res. 2012, 40, 4288-4297. [CrossRef]

64. Pompon, J.; Manuel, M.; Ng, G.K.; Wong, B.; Shan, C.; Manokaran, G.; Soto-Acosta, R.; Bradrick, S.S.; Ooi, E.E.; Missé, D.; et al. Dengue subgenomic flaviviral RNA disrupts immunity in mosquito salivary glands to increase virus transmission. PLoS Pathog. 2017, 13, e1006535. [CrossRef]

65. Livak, K.J.; Schmittgen, T.D. Analysis of relative gene expression data using real-time quantitative PCR and the 2- $\Delta \Delta C T$ method. Methods 2001, 25, 402-408. [CrossRef] [PubMed]

66. Reese, M.G. Application of a time-delay neural network to promoter annotation in the Drosophila melanogaster genome. Comput. Chem. 2001, 26, 51-56. [CrossRef]

67. Fornes, O.; Castro-Mondragon, J.A.; Khan, A.; Van Der Lee, R.; Zhang, X.; Richmond, P.A.; Modi, B.P.; Correard, S.; Gheorghe, M.; Baranašić, D.; et al. JASPAR 2020: Update of the open-Access database of transcription factor binding profiles. Nucleic Acids Res. 2020, 48, D87-D92. [CrossRef] [PubMed] 